\title{
Genres et compositeurs déclarés être écoutés en éducation musicale au collège, en France : enjeux didactiques et sociologiques
}

Genres and composers declared to be heard in music education at the Junior high school, in France: didactic and sociological issues

\section{Odile Tripier-Mondancin}

\section{OpenEdition Journals}

Édition électronique

URL : https://journals.openedition.org/educationdidactique/2168

DOI : 10.4000/educationdidactique.2168

ISBN : 978-2-7535-4191-7

ISSN : 2111-4838

Éditeur

Presses universitaires de Rennes

Édition imprimée

Date de publication : 20 mai 2015

Pagination : 81-105

ISBN : 978-2-7535-4146-7

ISSN : 1956-3485

Référence électronique

Odile Tripier-Mondancin, «Genres et compositeurs déclarés être écoutés en éducation musicale au collège, en France : enjeux didactiques et sociologiques », Éducation et didactique [En ligne], 9-1 | mai 2015, mis en ligne le 20 mai 2017, consulté le 16 août 2022. URL : http://journals.openedition.org/ educationdidactique/2168; DOI : https://doi.org/10.4000/educationdidactique.2168 


\section{GENRES ET COMPOSITEURS DÉCLARÉS ÊTRE ÉCOUTÉS EN ÉDUCATION MUSICALE AU COLLÈGE, EN FRANCE : ENJEUX DIDACTIQUES ET SOCIOLOGIQUES}

Odile Tripier Mondancin

Lécoute d'œuvres musicales est une activité essentielle de l'école généraliste française, collège compris. Étant donné la liberté de choix qu'ont les enseignants, qu'en est-il des répertoires déclarés être enseignés en collège ? Nous questionnons dans une visée descriptive-explicative, les genres et compositeurs déclarés être diffusés par des enseignants d'éducation musicale $(\mathrm{n}=104)$ dans quatre académies. Quelles sont les logiques qui conduisent ces enseignants à choisir tel ou tel genre musical, tel ou tel compositeur ? La méthode employée est l'enquête par questionnaire. Si des œuvres de toutes époques, du Moyen Âge à nos jours sont diffusées, c'est le répertoire tonal/ modal qui prédomine. Les œuvres diffusées le sont avant tout pour les notions musicales qu'elles véhiculent, en lien avec la faisabilité de leur perception. L'hypothèse selon laquelle on trouverait une influence réciproque significative entre l'ancienneté dans le métier, l'académie de provenance et certains choix est confirmée.

Mots-clés : transposition didactique, genres et compositeurs diffusés, éducation-musicale, logiques, variables explicatives.

Genres and composers declared to be heard in music education at the Junior high school, in France: didactic and sociological issues

Listening to musical works is a major activity in French general schools, junior high schools included. Given the freedom of choice of teachers, what about the repertoires which are declared to be taught in junior high schools? We question from a descriptive and explanatory viewpoint the genres and composers which are declared to be broadcast by music teachers $(n=104)$ in four regional education authorities. What logic leads these teachers to choose some musical genre or other, some composer or other? The method used is a questionnaire-based survey work. If it is true that musical works from all periods, from the Middle Ages up to the present day are broadcast, the tonal/modal repertoire prevails. The works are mainly broadcast for the musical notions they convey, linked with the feasibility of their perception. The hypothesis according to which there would be significant reciprocal influence between teachers seniority in their occupation, their regional education authority of origin and certain choices is confirmed.

Keywords: didactic transposition, broadcast genres and composers, musical education, logic, explanatory variables. 
Odile Tripier Mondancin

\section{INTRODUCTION}

Dès les instructions et programmes de 1938, l'enseignement de la musique dans l'enseignement secondaire généraliste français, positionne l'écoute et l'analyse d'œuvres comme une activité essentielle, aux côtés du chant (Tripier-Mondancin, 2008, 2010). Dans cet article, nous nous intéressons aux types d'œuvres écoutées en classe d'éducation musicale au collège, en France. L'étude équivalente, relativement aux répertoires chantés, a été menée en parallèle de celle-ci (Tripier-Mondancin, 2013b). En outre, des travaux menés par Maizières questionnent les mêmes objets musicaux à l'école primaire (accepté à l'évaluation, 2013).

L'absence d'étude sur ces sujets nuit cruellement à la connaissance de la forme scolaire de la culture musicale. Ce déficit conduit notamment la sociologie des pratiques culturelles à avoir tendance à oublier que les élèves sont confrontés à des savoirs musicaux, d'une manière générale à des répertoires, du début à la fin de leur scolarité obligatoire (soit, après la classe de $3^{\mathrm{e}}$ ) : "La musique ne faisant pas à proprement parler partie du socle commun de la culture scolaire, il s'agit en effet d'un domaine où l'on s'attend à voir jouer avec force l'influence des groupes primaires : environnement familial, groupe des pairs, communautés ethniques. » (Coulangeon, 2003, p. 4). Les résultats de l'enquête de Donnat menée en 1997 et sur lesquels s'adosse l'étude de Coulangeon, font toutefois le lien entre la pratique d'un instrument de musique plus élevée chez les plus jeunes de 15 à 19 ans (60\%) que chez les 25 à 34 ans et « les progrès continus de l'éducation musicale depuis plusieurs décennies dans le cadre scolaire et dans celui du temps libre $»$ (Donnat, 1998, p. 285-290). Pour autant, les pratiques vocales et l'écoute d'œuvres (qui nous occupe plus particulièrement dans cet article) sont négligées pour les premières et oubliées pour les secondes. Ces oublis, voire dénis (Coulangeon, 2003, p. 20-27) étaient déjà présents dans les travaux de Bourdieu : « [...] on ne saurait imputer à la seule action du système scolaire (et, à plus forte raison, à l'éducation artistique, de toute évidence presqu'inexistante, qu'il procurerait) la forte corrélation observée entre la compétence en matière de musique et de peinture [...] et le capital scolaire » (1979, p. 21). Mais les constats de Bourdieu, contrairement aux observations plus récentes s'expliquaient au regard des échantillons de la population française interrogés. En effet, ces générations scolarisées avant le collège unique (1975), n'avaient pas encore bénéficié des effets de la massification de l'enseignement secondaire. L'éducation artistique était loin d'être homogène : entre 1964 et 1970 , le nombre d'enseignants chargés d'enseigner la musique passa de 900 à 2612. Non seulement ils n'étaient pas suffisamment nombreux pour couvrir la demande sur tout le territoire français, mais encore, leur niveau de qualification était loin d'être majoritairement celui du CAEM, Certificat d'Aptitude à l'Éducation Musicale (Tripier-Mondancin, 2008, p. 173, 2010, p. 76-78). Bien entendu, ces oublis de variables n'invalident pas les résultats de l'étude de Coulangeon. En revanche, leur prise en compte nuancerait encore davantage les manipulations de variables ${ }^{l}$ et dès lors les analyses explicatives, dont celle du rôle joué aujourd'hui par l'école obligatoire, dont le collège.

Décrire les genres et compositeurs écoutés à partir des déclarations des enseignants d'éducation musicale, étudier le sens ou les raisons qu'ils donnent à leurs choix, puis, dans un troisième temps tenter d'expliquer ces choix grâce à certains traitements statistiques des données comme les analyses de variances, constituent dès lors les objectifs généraux de cette étude.

Cette étude s'impose d'autant que depuis les arrêtés de 1977-1978, les prescriptions concernant les compositions musicales à écouter se limitent à des cadres assez larges ; la contrainte d'une approche chronologique de la $6^{\mathrm{e}}$ à la $3^{\mathrm{e}}$, qui prévalut dans les instructions précédentes de 1938 à 1964, n'est plus à l'ordre du jour (Tripier-Mondancin, 2008, 2011, p. 127-128). Toutefois, le récent arrêté de Programmes de l'enseignement de l'éducation musicale de collège (MEN, 2008) cadre davantage les possibilités de choix : il s'agit de mettre chaque année en perspective au moins cinq œuvres écoutées et étudiées en profondeur, avec des « œuvres périphériques ", du point de vue des "domaines du timbre et de l'espace ", " de la dynamique ", « du temps et du rythme » " du successif et du simultané », « de la forme ", " des styles », en fonction de questions transversales. Le domaine des styles mentionne des « périodes historiques », des « aires géographiques et culturelles ». Sont mentionnées des grandes catégories musicales (savant/populaire, sacré/profane, descriptive, " pure », occidentale/non occidentale) et de nombreux genres musicaux (« chant grégorien, chansons, danses, concerto, opéra, oratorio, ballet, 
symphonie, lied, poème symphonique, comédie musicale », etc.) placés sur une échelle linéaire du temps. Il n'en demeure pas moins qu'aucun titre d'œuvre et aucun compositeur n'est cité en particulier.

Par conséquent, les enseignants d'éducation musicale demeurent en grande partie libres dans le choix des œuvres à enseigner. Cela signifie que si comme dans toute discipline, c'est à l'enseignant d'éducation musicale que revient le « traitement didactique du savoir » à enseigner, en savoir enseigné (Chevallard, 1991, p. 20), soit, la transposition didactique interne, il est aussi chargé d'une partie de la transposition didactique externe traditionnellement dévolue à la noosphère au sens chevallardien. Ce substantif parodique donné par Chevallard qualifie la sphère où l'on pense le fonctionnement didactique dans chaque discipline. C'est un « lieu» institutionnel placé entre ministère, société, université et école, dans lequel opèrent les groupes d'experts chargés de la conception des programmes (par exemple les Groupes Techniques Disciplinaires, ou GTD, à partir des années 1990). À quels objets de savoirs et pour quelles raisons, l'enseignant décide-t-il de confronter les élèves, au travers des répertoires qu'il choisit?

Alors que la notion de "culture commune", de « socle commun » au sein de l'école est affirmée (MEN, 2006), quatre questions émergent : 1. Qu'en est-il de la diversité et du type d'œuvres musicales écoutées en collège ? 2. En fonction de quels critères (et pour quelles raisons) sont-elles choisies par les enseignants? 3. Quelles variables peuvent expliquer ces choix ? 4. Pouvons-nous parler d'influence réciproque entre le contexte professionnel (académie, type de collège, nombre d'années dans le métier), la variable démographique (sexe) et les choix des enseignants ?

En l'absence d'étude systématique sur la question des répertoires écoutés, une enquête, à visée descriptive/compréhensive, sur la base d'observations empiriques, adressée à des enseignants d'éducation musicale de collège, se justifie pleinement dans le cadre approprié d'une démarche inductive/déductive, en ce qui concerne les questions 1,2 et 3 et hypothéticodéductive relativement à la question 4 .

En regard des travaux et théories disponibles, nous allons voir comment, les observations pédagogiques, les concepts de contenus d'enseignementapprentissage (Delcambre, 2007) plus précisément la distinction entre informations, connaissances et savoirs (Reboul, 1980, Astolfi, 1992, Develay, 1995), de transposition didactique (Chevallard, 1985, 1991, Bourg, 2008), de catégorisation du fait musical (Rice, 2003), peuvent aider à problématiser, à décrire, à expliciter les décisions de l'enseignant en matière de choix de répertoires voire, par inférence, de savoirs à enseigner/enseignés. La question de savoir si des répertoires fondés sur des listes de genres et de compositeurs mais aussi sur des critères et des raisons des choix peuvent être ou non considérés comme des indicateurs de savoirs à enseigner/ enseignés et non de connaissances sera discutée. Des variables explicatives des choix de l'enseignant seront envisagées.

La confrontation des résultats, avec (1) le modèle de la légitimité culturelle fondé sur le lien attesté « entre l'appartenance aux classes supérieures, le goût des arts savants et le rejet simultané des arts populaires » (Bourdieu, 1979 cité par Coulangeon, 2003, p. 4), avec (2) l'hypothèse, plus récente, fondée sur l'idée d'une « progression de l'éclectisme des goûts des classes supérieures, en particulier dans le domaine musical » (Peterson \& Simkus, 1992, cité par Coulangeon, 2003, p. 4), permettra d'élaborer de nouveaux questionnements et hypothèse.

\section{TRAVAUX ET THÉORIES POUR CONTRIBUER À LA DIDACTIQUE DE L'ÉCOUTE}

Toute démarche, y compris inductive, est dépendante d'une théorie, en amont de celle- $\mathrm{ci}^{2}$ (Chalmers, 1987, p. 50-72). Dans le cas présent, il s'agit davantage de constats pédagogiques que d'une théorie comme le signale J.-P. Mialaret dans un des rares articles en lien avec nos préoccupations (2001, p. 99). La synthèse des travaux scientifiques sur l'écoute musicale, qu'il proposait, montre qu'ils portaient exclusivement sur les dimensions psychologiques de l'écoute. En revanche de nombreuses observations pédagogiques faisaient état de deux grandes approches des situations scolaires d'écoute musicale : 1. La centration de l'enseignant sur la transmission du savoir à enseigner et 2. La centration des enseignants sur le désir, les besoins, la motivation des élèves qui conduit l'enseignant à faire des choix correspondants à ce qui est déjà apprécié. Nous tenterons de vérifier le degré d'acceptabilité de ces deux observations. 


\section{Des didactiques françaises à la didactique de l'écoute musicale}

La question des répertoires de genres et de compositeurs déclarés être écoutés en classe s'inscrit pour partie dans le questionnement des théories didactiques françaises, qui ont, selon Chevallard, à identifier et à désigner des « contenus de savoirs comme contenus de savoirs à enseigner » (1991, p. 39) et plus généralement « qui ont à décrire les formes complexes d'élaboration des savoirs scolaires, en référence aux disciplines, à leur histoire, à leur modes de constitution, à leurs fonctionnement dans les situations réelles d'enseignement " (Delcambre, 2007 , p. 49). Plus spécifiquement, ce questionnement s'inscrit dans la Didactique de la musique (pour davantage de précisions voir les articles de Bourg, 2008, 2011) et plus particulièrement encore, dans la Didactique de l'écoute. Même si les études de Mili (2011-2013) et de Villemin (2011) s'inscrivent pleinement dans ce domaine de recherche, elles ne concernent pas directement la question qui nous préoccupe.

En quoi des listes de genres et de compositeurs déclarés être écoutés en classe, ainsi que les critères et raisons qui ont présidé à leurs choix, relèveraientils d'une approche des savoirs à enseigner/enseignés, plus généralement des savoirs scolaires, d'autant que l'enquête se situe au niveau déclaratif et non pas effectif ? Même s'il y a de fortes chances pour que ce soit le cas, rien ne prouve a priori que « ce qui fait» critère de choix devienne dans la classe un objet de savoir enseigné au sens chevallardien de notion musicale issue de la transposition didactique (1991, p. 49). Rien ne prouve que la notion de compositeur ou de genre constituent des savoirs en soi. Les répertoires de genres et de compositeurs seraient a minima des véhicules ou des pourvoyeurs de savoirs. Ils ne seraient pas enseignés en tant que tels. Néanmoins, du fait de leur présence dans l'environnement de la classe en tant qu'objets musicaux diffusés, les élèves y seraient exposés, l'apprentissage pourrait se faire au moins de manière implicite, au sens donné par la psychologie cognitive (Musial, Pradère, Tricot, 2012, p. 22).

Il est donc nécessaire de rappeler ce qui peut être entendu par savoir, en regard de l'ensemble des objets musicaux.

\section{Du concept de savoir}

D'une manière générale, on rattache les savoirs et savoir-faire à la notion de contenu d'enseignement et d'apprentissage (Delcambre, 2007, p. 45). Epistémologiquement, relativement à la théorie des trois mondes de Popper (1978), à laquelle se réfère Astolfi (1994, p. 68-77), le savoir, en raison de son caractère objectivé (par un système théorique ; monde 3 qui renvoie aux contenus de pensée objectifs), institué (construit socialement et historiquement dans une institution), décontextualisé (de son lieu de production initial) et dépersonnalisé (certains savoirs n'ont plus d'auteur), se distinguerait, (1) de la connaissance (relative aux expériences subjectives du sujet, à ses représentations, en lien avec l'idée de naissance; monde 2) et (2) de l'information (objective ; monde 1 qui renvoie aux objets et aux états physiques), extérieure au sujet qui en prend connaissance, stockable, quantifiable, mise en forme pour circuler.

Chevallard introduit une nuance supplémentaire au sein des objets de savoir qui peuvent être explicitement ou implicitement construits dans l'enseignement : (1) les notions disciplinaires, dans notre cas musicales, issues de la transposition des savoirs savants (cf. infra), (2) les notions para-disciplinaires, dans notre cas les notions outils de l'écoute musicale, comme par exemple, les pratiques langagières spécifiques à la musique, qui doivent être connues de l'élève parce que nécessaires, mais qui ne sont pas enseignées selon Chevallard et (3) les notions proto-disciplinaires comme la reconnaissance des conditions d'emploi des notions musicales, qui vont de soi et ont donc un statut implicite y compris pour les enseignants (1991, p. 51).

Cette conceptualisation, de ce qui caractérise le savoir au sens large, ne s'oppose pas à celle élaborée depuis longtemps par la philosophie de l'enseignement mais aussi par la psychologie. La distinction savoir, savoir-faire, savoir-être présente dès 1980 chez un philosophe de l'éducation comme Reboul (2010) oppose trois formes d'apprentissage, " apprendre que » (informations, renseignement) et « apprendre à » (savoir-faire), apprendre (étude, compréhension), apprendre à être. Pour lui « apprendre que » et " apprendre à » sont étroitement liés : « il n'existe pas d'activité purement physique et tout savoir-faire depuis nager jusqu'à raisonner, intéresse l'homme tout entier »(ibid. p. 67). On 
Odile Tripier Mondancin

retrouve cette intrication savoir/savoir-faire au sein de la notion unitaire de praxéologie, chez Chevallard. Même si ces nuances ont été réduites chez les théoriciens de l'intelligence artificielle, à une opposition entre connaissances déclaratives (savoir que) et connaissances procédurales (savoir-faire), une des théories de la connaissance (qui ne s'en tient pas à la programmation de machines) au sein de la psychologie cognitive (Anderson \& al, 2001, Tricot, 2011) y articule la dimension du général au particulier. Sans entrer dans les détails des différents formats de connaissances qui en découlent, précisons, que dans ce cadre, le savoir-faire est également considéré comme une combinaison de séquences d'actions physiques et/ou d'opérations mentales.

Dans le cadre d'une didactique de choix des œuvres musicales à écouter, par exemple, il peut être intéressant d'observer si les œuvres écoutées en classe sont rattachées systématiquement à leur compositeur : dans ce cas, le compositeur se fait-il savoir enseigné ou reste-t-il à sa place initiale d'auteur du savoir enseigné qu'est l'œuvre; quel statut l'enseignant prévoit-il de donner à la notion de compositeur : information fournie/non fournie à l'élève ? Connaissance de l'enseignant ? Savoir construit voire théorisé ? Est-ce que le compositeur de l'œuvre se situe au même niveau que l'auteur de la mécanique classique qu'était Isaac Newton et dans ce cas est-il même mentionné ou bien l'œuvre s'en trouve-t-elle dépersonnalisée? En d'autres termes, le statut ou l'état de l'objet de savoir « compositeur» se transforme-t-il en rentrant dans la classe? De la même manière, la notion de genre auquel appartient une œuvre est-il enseigné en tant que système théorique qui permet de classifier l'œuvre, auquel cas, en devient-il savoir enseigné ?

\section{Transposition didactique}

Au-delà du statut des connaissances, des savoirs (au sens large), des informations, faire le choix d'un répertoire de genres et de compositeurs selon des critères, c'est également travailler « à la transposition didactique » ou « dans la transposition didactique " (Chevallard, 1991, p. 18). Ce concept issu de la sociologie (Verret, 1975), théorisé par un didacticien des mathématiques (Chevallard, 1985), puis mis à l'épreuve par de nombreux didacticiens, a été assez récemment importé dans la didactique musi- cale dans une démarche comparatiste et critique par Bourg $(2008,2011)$. « La transposition didactique a lieu quand les éléments du savoir passent dans le savoir enseigné » (Chevallard, 1991, p. 22). Nous considérons, à l'instar de Bourg, que « c'est avant tout un outil heuristique aidant à la modélisation des phénomènes didactiques » (2008, p. 70). Comme l'analyse Chevallard, c'est à la noosphère que revient « la sélection des éléments du savoir savant ${ }^{3}$ qui, désignés par là comme "savoirs à enseigner", seront alors soumis au travail de la transposition ; c'est elle, encore, qui va assumer la partie visible de ce travail, ce qu'on peut appeler le travail externe de la transposition didactique, par opposition au travail interne, qui se poursuit, à l'intérieur même du système d'enseignement $[\ldots] »(1991$, p. 30-31). Comme nous l'avons vu les enseignants prolongent en quelque sorte le travail fait par les rédacteurs de programmes.

Nous posons l'hypothèse que parce qu'il y a choix et dès lors prélèvement d'objets de savoir (par exemple, des genres musicaux, des compositeurs), selon des critères précis (par exemple en fonction de notions musicales), en fonction de raisons énoncées, un premier phénomène de transposition s'observe. Il reste à analyser ce qui est de l'ordre des savoirs enseignés ou non.

Ce concept permet donc d'identifier, pour les analyser, les répertoires présents dans la classe et de montrer comment, en relevant de la transposition externe, les répertoires écoutés à l'école et au collège sont choisis et permettent d'inférer peut être des savoirs enseignés. Dans le cadre de cette recherche, nous questionnons le lien entre l'élaboration du corpus, au sens de prélèvement-sélection fait par l'enseignant, au sein de répertoires de références et les critères de choix et non pas la transformation subie par le savoir, dès lors qu'on l'enseigne.

\section{Origine des connaissances des enseignants d'éducation musicale}

Une enquête menée en 2005 montrait que $94 \%$ des enseignants d'éducation musicale de l'échantillon ( $\mathrm{n}=191)$ ont suivi une licence de musiquemusicologie avant d'entrer dans le métier (TripierMondancin, 2008, p. 322-323). On peut dès lors faire l'hypothèse qu'en ce qui concerne les œuvres ou compositions musicales à faire écouter aux élèves, une des sources des connaissances et donc des 
savoirs de référence de l'enseignant relève donc en grande partie du produit de la science humaine qualifiée de musicologie, en France (1951), en lien avec le programme fondateur de la Musikwissenschaft en Allemagne. À partir de 1954, l'ethnomusicologie vint s'adosser à la musicologie. Notons que les musiques populaires ont été assez récemment introduites dans les études musicologiques de type universitaire.

Dans ces deux cadres universitaires sont élaborés des corpus d'œuvres, des principes de catégorisation diachroniques, synchroniques, stylistiques ainsi que des méthodes d'analyse.

Dès leur plus jeune âge (moyenne 8 ans, médiane, 7 ans), $97 \%$ de ces enseignants ont suivi un parcours dans des écoles nationales de musique ou des conservatoires nationaux de région, (Tripier-Mondancin, 2008, p. 289) : c'est sans doute une des premières sources potentielles de savoirs de référence et que Johsua qualifie de savoirs experts (Johsua, 1997) soit, en général, le solfège, les pratiques instrumentales et vocales, l'écriture.

Au-delà des liens que les enseignants entretiennent avec ces deux grandes institutions (l'université, le conservatoire pour résumer), ils assistent à des concerts, des spectacles, possèdent un grand nombre de supports de musique enregistrée (éditeurs, diffuseurs, arts du spectacle), mais également de partitions (éditeurs), tout en bénéficiant des apports d'internet ; ils continuent à se former (formation continue, secteur associatif).

Ainsi ces enseignants sont confrontés à un grand nombre de sources de savoirs musicaux de référence.

\section{Délimitation de la recherche}

Dans le cadre de cette étude exploratoire, devant l'ampleur des possibilités de choix, nous avons volontairement restreint la recherche aux genres et compositeurs déclarés être diffusés en classe, sans avoir la preuve qu'ils le sont réellement et qui plus est, en tant qu'objets de savoir explicites. C'est l'idée d'inventaire méthodique le plus systématique possible des genres et compositeurs écoutés qui a orienté le choix de ce cadre d'étude. C'est ainsi que le terme répertoire s'est imposé. En effet, la liste des genres musicaux et des compositeurs reste mesurable, contrairement aux œuvres qui sont en nombre quasi infini. Les listes de genres musicaux ont en outre l'avantage de permettre de « remonter » par tris successifs, à des niveaux taxinomiques plus globaux comme la musique sacrée, profane, instrumentale, vocale, européenne, extra-européenne, voire savante, populaire, traditionnelle.

Le concept de genre est un paradigme utilisé à la fois dans les sciences de la nature et dans l'ensemble des sciences humaines. Il correspond à un niveau de classification ou de taxinomie mais aussi de regroupement d'objets sur la base de similitudes. Il peut être regroupé lui-même en des catégories plus générales. Pour Dahlhaus, dans le dictionnaire Science de la musique dirigé par Honegger, « les noms des différents genres musicaux ne dépendent pas en général de la forme mais de la destination de l'œuvre pour les voix ou pour les instruments (cantate, sonate), de la formation et du nombre des parties (quatuor à cordes), de la technique d'écriture (fugue), du texte (messe), de la fonction d'une œuvre (prélude), ou du lieu de son exécution ("sonata da chiesa") » (1976, p. 396-397). Denizeau, écrit plus récemment : "le genre nomme l'objet musical, le distingue aux oreilles de l'auditeur, cependant que la forme l'organise, lui donne une vie cohérente $»(1997$, p. 5). Le niveau de catégorisation pour classer les œuvres entre elles, que constitue le genre, fait dès lors consensus en musicologie. L'analyse des ouvrages aux éditions Minerve intitulés Vocabulaire de... (cf. bibliographie) consacrés aux termes qui permettent de décrire la musique par époque, témoignent de cette stabilité. Les genres musicaux se regroupent entre eux en écoles (École Notre-Dame, École de Vienne) ou en grandes catégories (musique sacrée, musique religieuse, musique profane, musique de chambre, genres vocaux, genres instrumentaux, musique pour piano ou pour orchestre). Ces différents niveaux de catégorisation témoignent de la manière dont l'objet est regardé, analysé.

Le concept de courant - très proche de celui de genre - est davantage utilisé à propos de la musique du $\mathrm{XX}^{\mathrm{e}}$ siècle (musique concrète, musique spectrale, sérialisme, dodécaphonisme, minimalisme, be-bop, free-jazz, fusion) (Bras, 2003, p. 10). Dans cette étude, nous entendrons par genre, plus généralement, école et courant musical : ces trois concepts fondent l'enquête.

Enfin, même si la distinction populaire/savant/ traditionnel fait l'objet de ce que nous qualifions une crise paradigmatique au sein de la musicologie, nous l'utilisons car elle permet non seulement de synthétiser la pensée mais également de faire le lien avec les travaux de la sociologie des pratiques culturelles. 
Odile Tripier Mondancin

À l'instar de Rice, nous entendons par répertoire savant, tout répertoire musical écrit (2003). Nous préfèrerons ce terme à celui de « classique » auquel est adjoint celui "d'opéra » que les sociologues emploient, mais qui est trop imprécis en regard de la didactique musicale.

\section{Sociologie des genres musicaux écoutés}

Dès lors, les travaux de Coulangeon nous intéressent particulièrement car ils portent sur «les genres musicaux » écoutés en tant " qu'approximation satisfaisante des préférences » (2003, p. 9).

La théorie bourdieusienne et les travaux empiriques qui ont suivi attestent d'un lien significatif entre les préférences esthétiques et le statut socioprofessionnel, l'origine sociale et le capital culturel amassé, en lien avec les études supérieures, entre autres (Bourdieu 1979, Di Maggio et Mohr, 1985 cités par Coulangeon, 2003, p. 4). Ainsi, le lien entre, le fait d'appartenir aux classes sociales supérieures et la préférence pour la musique savante tout en rejetant les musiques populaires est attesté.

Depuis les années 1990, ces conclusions sont infléchies par des travaux américains et confirmées par les travaux français, qui mettent en évidence un éclectisme des goûts des classes supérieures (données datant de 1982 puis de 1992), alors que les classes populaires seraient des amateurs exclusifs (mais cela reste à nuancer) de variétés. Cette transformation s'interprète comme un « recul des frontières dressées entre les groupes sociaux » (Peterson et Simkus, 1992 cités par Coulangeon, p. 6). Les thèses postmodernes avancent même que ce sont la production industrielle des biens symboliques et la société des loisirs qui font « perdre aux élites culturelles le monopole des normes et des échelles de valeur esthétique, au profit de la coexistence d'une pluralité d'échelles de jugements, d'une "invasion démocratique" du monde des arts (Michaud, 1997) et qui mettent en cause le modèle unificateur de la légitimité culturelle qui est au principe des phénomènes de domination symbolique décrits par Pierre Bourdieu (Featherstone, 1995) » (cité par Coulangeon, p. 6). Mais Van Eijck (1997, cité par Coulangeon, p. 7) avance l'idée que l'éclectisme des goûts serait lié à l'élargissement de la base sociale du recrutement des classes supérieures. En outre, les élites ne restent pas indifférentes aux distinctions, qui reposent toujours sur les objets culturels mais élargis (au jazz par exemple et plus récemment au rock) et sur la manière de les consommer (cf. cas du jazz et de la tradition afro américaine qui font l'objet d'une « esthétisation de l'écoute au sein des milieux intellectuels » (ibid. p. 7) par opposition aux usages plus populaires relevant par exemple de la danse. La domination symbolique continue de s'exercer mais sous une autre forme. Cinq profils de préférences, que nous résumons sous la forme du tableau 1 ,

Tableau 1. Comparaison des profils de l'étude de Coulangeon avec le profil des enseignants d'éducation musicale (Tripier-Mondancin, 2008)

\begin{tabular}{|c|c|c|c|c|c|}
\hline $\begin{array}{l}\text { Coulangeon ( } 2003 \text { ) / } \\
\text { écoute, préférences } \\
\text { des français }\end{array}$ & $\begin{array}{l}\text { I - Classes } \\
\text { supérieures plus de } \\
40 \text { ans, revenus } \\
\text { élevés, diplômés, } \\
\text { composante } \\
\text { féminine }\end{array}$ & $\begin{array}{l}\text { II - Plus de } 60 \text { ans, } \\
\text { revenu, statut } \\
\text { socioprofessionnel et } \\
\text { diplôme? }\end{array}$ & $\begin{array}{l}\text { III - Moins de } 25 \text { ans, } \\
\text { statut } \\
\text { socioprofessionnel ? et } \\
\text { classes supérieures, } \\
\text { composante masculine }\end{array}$ & $\begin{array}{l}\text { IV - Classes } \\
\text { populaires, } \\
\text { employés ouvriers }\end{array}$ & $\begin{array}{l}\mathrm{V} \text { - Plus de } 60 \text { ans, } \\
\text { générations } \\
\text { antérieures à la } \\
\text { banalisation de la } \\
\text { musique } \\
\text { enregistrée }\end{array}$ \\
\hline & $\begin{array}{l}20 \% \text { de } \\
\text { l'échantillon : trois } \\
\text { genres savants, } \\
\text { dont jazz }\end{array}$ & $\begin{array}{l}\text { 13\% de l'échantillon: } \\
\text { Musique d'ambiance, } \\
\text { de danse, folklorique, } \\
\text { de film opérette }\end{array}$ & $\begin{array}{l}8 \% \text { : Rap rock, hard } \\
\text { rock, musiques du } \\
\text { monde, variétés } \\
\text { internationales }\end{array}$ & $\begin{array}{l}45 \%: \text { forte } \\
\text { spécialisation des } \\
\text { préférences (un } \\
\text { genre cité), attrait } \\
\text { pour les variétés }\end{array}$ & $15 \%$ : aucun genre \\
\hline $\begin{array}{l}\text { Tripier-Mondancin } \\
\text { (2008, p. } 384) / \\
\text { pratique } \\
\text { instrumentale, vocale, } \\
\text { écoute d'œuvres par } \\
\text { les enseignants d'EM } \\
\text { interrogés, en 2005- } \\
2006\end{array}$ & \multicolumn{4}{|c|}{$\begin{array}{l}\text { Répertoire savant }>\text { populaire }>\text { traditionnel : (dans l'ordre décroissant) classique, baroque, } \\
\text { romantique, chanson française, } 20^{\mathrm{e}} \text { sav. avant 1945, Renaissance, chanson anglo-saxonne, } 20^{\mathrm{e}} \\
\text { savant après } 1945 \text {, jazz, var. internationales, Moyen Âge, trad. Amérique du Sud, Breton, occitan, } \\
\text { monde arabe Irlande, Europe de l'Est, Amérique du Nord, Afrique, caraïbes, courant } \\
\text { électroniques... }\end{array}$} & \\
\hline $\begin{array}{l}\text { Ibid. préférences, p. } \\
492\end{array}$ & \multicolumn{4}{|c|}{$\begin{array}{l}\text { Répertoire savant (Moyen Âge au XIXe) > populaire }>\text { traditionnel }>\text { jazz }>\text { XX } X^{\mathrm{e}} \text { savant }>\text { musique } \\
\text { de film }\end{array}$} & \\
\hline
\end{tabular}


émergent de l'étude de Coulangeon. Dès lors, référés à cette étude et en raison de leur éclectisme, les enseignants d'éducation musicale de l'échantillon d'une autre enquête menée en 2005 ( $\mathrm{n}=191$ ) (TripierMondancin, 2008) ne se situeraient dans aucune de ces catégories en particulier. Ils se situeraient dans toutes et bien au-delà, en dehors de la cinquième qui ne fait référence à aucun genre musical.

Quant aux résultats de l'étude des groupes socioprofessionnels d'appartenance des pères et mères des enseignants du même échantillon, comparés à ceux de la population française des actifs, ils montrent que ces enseignants sont issus très majoritairement de classes sociales favorisées : les cadres, les professions supérieures et les artisans, sont surreprésentés, tandis que les professions intermédiaires, les employés, les agriculteurs et les ouvriers sont sousreprésentés. Quant à leurs ascendants, ils pratiquent la musique trois fois plus que la moyenne nationale, tant côté père que mère (Tripier-Mondancin, 2008, p. 271-274).

\section{DÉMARCHE, MÉTHODOLOGIE DE RECHERCHE}

Nous n'avons pas souhaité poser d'hypothèse à propos des types d'œuvres déclarées être écoutées et analysées, durant les cours d'éducation musicale, ou encore, à propos des raisons ou critères qui amènent les enseignants à faire ces choix. En effet, les possibilités sont trop nombreuses pour qu'une hypothèse générale, si elle était confirmée, arrive provisoirement à répondre de manière synthétique aux questions sous-jacentes. En outre les recherches de type descriptives/compréhensives n'étant pas pléthoriques sur le sujet, nous avons donc délibérément décidé d'inscrire cette recherche dans une démarche fondée, en grande partie, sur l'induction/déduction de règles ponctuellement justes. Quelques hypothèses précises ont été formulées selon lesquelles nous pouvons parler d'influence réciproque entre les quatre variables indépendantes explicatives (genre, ancienneté dans le métier, académie au moment de l'enquête et type de collège, soit ZEP ou RAR, CHAM, ordinaire) et les variables à expliquer : genres et compositeurs retenus, logique des choix.

Nous voulions pouvoir recueillir un nombre de données qui autorise l'élaboration d'un point de vue général sur cette activité, selon une forme de représentativité. C'est donc le questionnaire, qui a été choisi comme outil de recueil de données. Il a été élaboré et mis en ligne sur Internet à l'aide de l'application Lime Survey ${ }^{\odot}$. Avec l'accord des IA-IPR (Inspecteurs d'Académie, Inspecteurs Pédagogiques Régionaux), que nous remercions, les correspondants TICE musicaux des quatre académies se sont chargés de la diffusion de l'adresse internet. Un questionnaire permet de faire un relevé de données sur un temps d'enseignement long (les quatre ans du collège) alors que des observations filmées, si elles permettent d'observer les savoirs réellement enseignés, limitent la perception de ces savoirs au temps de la classe observée.

Dans un souci d'évitement des approximations ou des oublis, tout autant que de standardisation de la passation et d'efficacité de traitement des réponses (estimer des grandeurs), nous avons proposé des questions en éventail fermé complétées par des questions ouvertes. Les questions en éventail permettent de générer des statistiques descriptives (fréquences, taux, rangs de moyenne) et inférentielles (Anova). Les questions ouvertes permettent d'élaborer des taxinomies grâce à un travail de catégorisation des réponses, par analyse du texte puis codage : méthode que vous validons par un accord intra-juge (par test/ retest). Le processus de catégorisation consiste à répartir des éléments dans des classes. L'exemple le plus représentatif d'une catégorie est retenu pour l'analyse. L'ensemble des données est saisi puis traité à l'aide du logiciel d'analyse statistique SPSS (Kinnear, Gray, 2005)

À la suite des questions relatives aux quatre variables indépendantes déjà citées, il s'agissait pour les enseignants :

De classer par ordre décroissant les 13 critères proposés qui les amènent à diffuser telle ou telle composition en classe (question en éventail ouvert) : « Lorsque vous choisissez les œuvres ou compositions à faire écouter/ analyser à vos élèves, vous le faites plutôt en fonction de... ». La liste de ces 12 critères (tableau 5) avait été élaborée grâce à la méthode $\mathrm{du}$ focus group (entretien de groupe), lors de stages de formation continue. La terminologie liée spécifiquement à la récente publication des programmes de 2008, au moment de l'enquête, a volontairement été laissée de côté, afin d'éviter les phénomènes de désirabilité. De cocher, parmi une liste de genres, de courants, d'écoles et de compositeurs, classés par période, par souci de systématisme, dans un 
ordre chronologique (Moyen Âge, Renaissance, Baroque, Classique (1760-1810), Romantique (1813-1890), XX et du XXI ${ }^{\mathrm{e}}$ savant moderne et post-moderne, $\mathrm{XX}^{\mathrm{e}}$ et du XXI ${ }^{\mathrm{e}} \mathrm{jazz}$, populaire (variété française, pop, rock et dérivés, variété anglo-saxonne, électronique) et par aire musicale géographique, celles et ceux qui sont écoutés en classe (question en éventail ouvert).

Ce niveau de détail, par période et par aire géographique, à propos du répertoire savant a été adopté pour éviter un effet de nomenclature sur les résultats, que l'on peut trouver dans les enquêtes sociologiques (Donnat, 1998, Coulangeon, 2010, p. 57).

La même question était ainsi posée à plusieurs reprises: " quels sont les genres musicaux du (Moyen Âge) que vous faites écouter aux élèves, de la $6^{\mathrm{e}}$ à la $3^{\mathrm{e}}$ ? ». La même question était doublée à propos des compositeurs. Quant à la question relative aux cultures musicales traditionnelles, elle était posée différemment : en lieu et place des périodes, elle proposait des aires géographiques détaillées par pays pour l'Europe (par exemple, traditionnel français, italien, espagnol...).

C'est en croisant différents ouvrages musicologiques et ethnomusicologiques, que nous avons constitué les listes les plus complètes des genres et des compositeurs pour une période donnée, ou des aires géographiques pour un continent donné (Chailley, 1958, Massin, 1985, Bosseur, 1996, Denizeau, 1997, 2010, Goubault, 1997, 2000, Ferrand, 1999, Lemaître, 1992, Michels, 1985, Aubert, 2003, Bras, 2003, Canguilhem, Ferrand, 2005, Noiray, 2005, Von der Weidt, 1992). La question du rattachement du jazz, au «populaire » ou au « savant » fait encore débat aujourd'hui au sein de la musicologie suivant les différents courants de jazz et y compris au sein de la production d'un même musicien. La sociologie considère qu'il a été "dignifié » par les catégories sociales supérieures. Il importait que, dans tous les cas, le jazz apparaisse en tant que tel, pour des raisons de lisibilité. Le jazz a par conséquent été placé, dans la plupart des questions, tantôt aux côtés de la catégorie des musiques populaires du $\mathrm{XX}^{\mathrm{e}}$ siècle (pour le jazz d'avant-guerre), tantôt aux côtés du répertoire savant. Dans les tableaux récapitulatifs de résultats, nous l'avons fait figurer dans la colonne du $\mathrm{XX}^{\mathrm{e}}$ avec les musiques populaires.

Enfin, chaque question, relative aux genres musicaux écoutés par époque, était suivie par une ques- tion sur les raisons pour lesquelles ces genres étaient diffusés (question ouverte).

Nous souhaitions pouvoir croiser par triangulation, ces nouvelles données avec celles recueillies à la première question en éventail ouvert (critères de choix à hiérarchiser et critères ouverts).

Pour des raisons de temps de passation, il ne s'agissait pas d'entrer dans le détail des niveaux auxquels tel répertoire est écouté-analysé, encore moins de demander le titre des œuvres (la liste en aurait été trop longue sinon très incomplète).

Sur les quatre académies dans lesquelles l'enquête a été proposée via les listes de courriels académiques (Bordeaux, Toulouse, Clermont-Ferrand, Limoges), 116 enseignants ont répondu pour une population de 1020 professeurs en poste (public et privé sous contrat réunis) dans ces quatre académies, en 2010, année de l'enquête. Nous avons pu exploiter 104 formulaires de réponses. Le taux de réponse est de $10,5 \%$ ce qui confère à notre échantillon de type non probabiliste (fondé sur un appel au volontariat des participants), une représentativité exemplaire (Dépelteau, 2003, p. 230).

\section{RÉPERTOIRES ÉCOUTÉS AU COLLÈGE : GRANDES TENDANCES, PREMIÈRES DISCUSSIONS}

\section{Très grande diversité, au profit du savant}

L'addition des fréquences des genres et des compositeurs cochés par les enseignants comme étant écoutés en classe de la $6^{\mathrm{e}}$ à la $3^{\mathrm{e}}$, en lien avec le calcul de la moyenne des réponses cochées (tableau 2) nous permet de comparer les grandes tendances par période. Les résultats montrent que des œuvres appartenant à toutes les époques et esthétiques, du Moyen Âge à nos jours, sont diffusées sans discontinuité, mais dans des proportions différentes.

Trois catégories de genres sont déclarées plus nettement écoutées que les autres : les genres populaires additionnés au jazz (389), les genres savants de l'époque baroque (386), ceux du Moyen Âge (367). En revanche, trois le sont beaucoup moins : dans l'ordre décroissant, ceux du XX $\mathrm{X}^{\mathrm{e}}$ issus d'une esthétique savante (243), les genres issus des cultures traditionnelles (220), ceux de la Renaissance (les moins diffusés, 195). Enfin, Les genres classiques (318) et romantiques (322) sont moyennement diffusés. 
Tableau 2. Nombre de genres musicaux et de compositeurs proposés dans le questionnaire et cochés par les enseignants $(n=109)$

\begin{tabular}{|c|c|c|c|c|c|c|c|c|c|}
\hline $\begin{array}{l}\text { Décompte des } \\
\text { réponses pour } \\
n=109, \\
\text { rapport à la } \\
\text { moyenne }\end{array}$ & $\begin{array}{c}\text { Moyenne } \\
\mu\end{array}$ & $\begin{array}{l}\text { Moyen } \\
\text { Âge }\end{array}$ & Renaissance & Baroque & $\begin{array}{c}\text { Classique } \\
(1760- \\
1810)\end{array}$ & $\begin{array}{c}\text { Romantique } \\
(1813- \\
1890)\end{array}$ & $\begin{array}{l}\text { XXe XXIe } \\
\text { savant : } \\
\text { modern } \\
\text { e et } \\
\text { postmo } \\
\text { derne }\end{array}$ & $\begin{array}{c}\text { XX } X^{\mathrm{e} X I e} \\
\text { jazz et } \\
\text { populaire }\end{array}$ & $\begin{array}{l}\text { Cultures } \\
\text { traditionn } \\
\text { elles / } \\
\text { aire géo. }\end{array}$ \\
\hline $\begin{array}{c}\text { Genres ou aires } \\
\text { proposés }\end{array}$ & 28,5 & $26<\mu$ & $18<\mu$ & $39>\mu$ & $26<\mu$ & $38>\mu$ & $28<\mu$ & $28<\mu$ & $25<\mu$ \\
\hline Genres cochés & 303,25 & $367>\mu$ & $195<\mu$ & $386>\mu$ & $318>\mu$ & $322>\mu$ & $243<\mu$ & $389>\mu$ & $220<\mu$ \\
\hline $\begin{array}{l}\text { Pourcentage de } \\
\text { genres cochés }\end{array}$ & & $15 \%$ & $8 \%$ & $17 \%$ & $13 \%$ & $13 \%$ & $10 \%$ & $15 \%$ & $9 \%$ \\
\hline $\begin{array}{c}\text { Courants, } \\
\text { écoles, } \\
\text { compositeurs } \\
\text { proposés }\end{array}$ & 22,6 & $12<\mu$ & $18<\mu$ & $18<\mu$ & $12<\mu$ & $30>\mu$ & $46>\mu$ & $\begin{array}{l}\text { Question } \\
\text { ouverte }\end{array}$ & ---- \\
\hline $\begin{array}{c}\text { Compositeurs } \\
\text { cochés }\end{array}$ & 286,67 & $212<\mu$ & $165<\mu$ & $341>\mu$ & $87<\mu$ & $457>\mu$ & $458>\mu$ & ---- & ---- \\
\hline $\begin{array}{c}\text { Pourcentage de } \\
\text { compositeurs } \\
\text { cochés }\end{array}$ & & $12 \%$ & $10 \%$ & $20 \%$ & $5 \%$ & $26 \%$ & $27 \%$ & & \\
\hline
\end{tabular}

En ce qui concerne les compositeurs savants choisis, ce sont très nettement les compositeurs du $\mathrm{XX}^{\mathrm{e}}$ siècle (458) et ceux issus du mouvement romantique (457 occurrences) qui sont les plus retenus. Les compositeurs de la Renaissance sont parmi les moins diffusés mais ce seraient les classiques qui le seraient encore moins. Les compositeurs et/ou écoles du Moyen Âge sont également en dessous de la moyenne de l'ensemble des genres diffusés. Nous n'avons pas proposé de liste de compositeurs appartenant aux catégories de musiques populaires du $\mathrm{XX}^{\mathrm{e}}$ et $\mathrm{XXI}^{\mathrm{e}}$ et jazz car la liste aurait été trop longue. Il en est de même pour les compositeurs des cultures traditionnelles, qui, la plupart du temps, restent anonymes. Il est dès lors impossible de comparer le poids des compositeurs diffusés toutes catégories confondues. Malgré tout, il est très clair que le répertoire savant est beaucoup plus écouté que le répertoire populaire et traditionnel additionnés.

Si nous comparons ces résultats avec ceux issus d'une enquête menée durant notre thèse (2005-2006) et fondée, entre autres, sur les pratiques, observées et filmées cette fois, de 23 enseignants (ces observations furent suivies par des entretiens d'explicitation), on constate que les grandes tendances sont similaires. Par exemple, sur 29 titres, les œuvres diffusées témoignent $\mathrm{du}$ fait que le répertoire populaire et savant (dont jazz) du XX $X^{e}$ siècle est majoritairement diffusé (12/29) : trois gospels, trois chansons, deux standards de jazz, un blues, l'introduction de L'oiseau de feu de Stravinsky, The Young Person's Guide to the Orchestra, op. 34 de Britten, un extrait de comédie musicale.

\section{Première discussion}

Rien n'indique si les analyses qui suivent, référées aux travaux de la musicologie historique, soustendent de la part des enseignants interrogés, une volonté explicite d'enseigner ces différents aspects, ces différents équilibres. En revanche ces analyses peuvent servir de point d'appui à une réflexion sur les enjeux d'une didactique de l'écoute musicale, en termes de savoirs à enseigner au sens large.

\section{Les ouvres $d u X X^{e}$ siècle proches du temps des élèves}

Tout en rappelant que nous n'avons pas questionné les compositeurs des musiques populaires, jazz et des cultures traditionnelles, si l'on regroupe les esthétiques toutes époques et toutes méta-catégories confondues (savantes, populaires, traditionnelles), alors les œuvres du XXe apparaissent comme les plus largement diffusées (figure 1). Ce sont aussi celles qui sont les plus proches des élèves, dans le temps.

\section{Avènement du statut du compositeur au XIX et au $X X^{e}$}

Dans la catégorie des musiques "savantes ", la prédominance du nombre de compositeurs diffusés de l'époque romantique et du $\mathrm{XX}^{\mathrm{e}}$ siècle peut s'expliquer par l'avènement, durant le XIX ${ }^{\mathrm{e}}$ siècle, du statut 
Figure 1. Somme des genres et compositeurs diffusés par esthétique ( $\mathrm{n}=104$ enseignants)

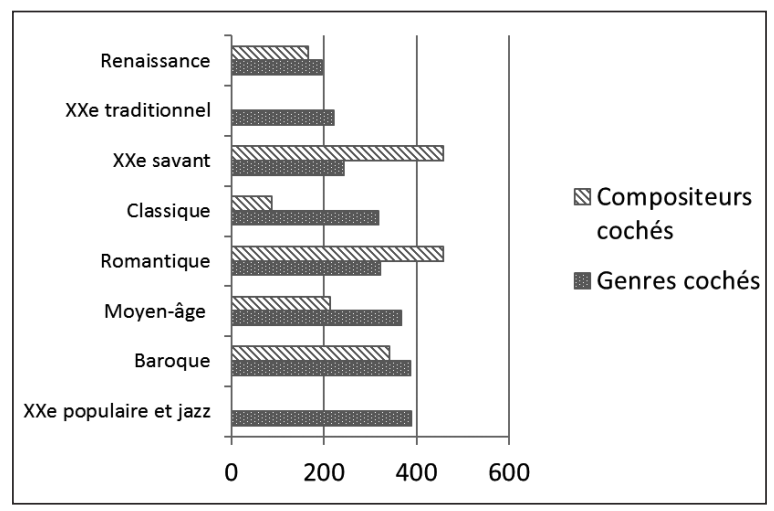

du compositeur, la mise sur piédestal de son expression mais aussi de celle de l'interprète (Heinich, 2005), ou encore, par le foisonnement et la singularité, à partir du $\mathrm{XX}^{\mathrm{e}}$ siècle, des individualités.

\section{Musique tonale-modale}

D'un point de vue stylistique, au sens dans lequel Goodman le définit, soit, la manière d'écrire (2001, p. 35-50), c'est la musique tonale-modale qui est la plus diffusée.

\section{Les genres profanes plus diffusés que les genres sacrés suivant les époques}

Un tri des réponses (à partir des tableaux 3 et 4) montre que les genres profanes sont plus diffusés que les genres sacrés ou religieux. Toutefois, pour les périodes du Moyen Âge ${ }^{4}$, de la Renaissance ${ }^{5}$ et de l'époque baroque ${ }^{6}$, la tendance est inverse en faveur des musiques sacrées. Aucun genre sacré ou religieux du XIXe et du XXe siècle ne semble retenir l'attention, si ce n'est du côté de ce que Caron qualifie de « chant religieux populaire $»$ (Caron, 2003, p. 57), soit, les negro spirituals et les gospels ( $28 \%$ ).

\section{Les genres instrumentaux presque autant diffusés que les genres vocaux}

Un nouveau tri montre que toutes époques confondues, les genres vocaux à la fois profanes et sacrés (1057 citations) sont un peu plus diffusés que les genres instrumentaux (906 citations). Mais ce sont surtout les genres vocaux du Moyen Âge et de la Renaissance qui marquent la différence. Une explication pourrait être liée au fait que c'est la présence de musique vocale, et non pas instrumentale, qui est attestée dans les livres liturgiques bien avant que le premier livre de musique ne soit édité en 1501 à Venise, par Petrucci. La notation musicale, les différents types de traités, d'écrits sur la musique ainsi que les premiers livres de musique sont au service avant tout de la liturgie et dès lors de la musique vocale (Massip, p. 22). Cet état de faits peut aussi expliquer le résultat précédent en ce qui concerne la prédominance de la diffusion du répertoire sacré sur le répertoire profane du Moyen Âge et de la Renaissance.

Les genres instrumentaux de l'époque baroque ${ }^{7}$ (194) sont autant diffusés que les vocaux ${ }^{8}$ (192); une inversion s'opère relativement aux $\mathrm{XVIII}^{\mathrm{e} 9}$ et $\mathrm{XIX}^{\mathrm{el0}}$ siècles : les genres instrumentaux sont davantage diffusés que les vocaux.

Les choix des enseignants semblent refléter les grandes évolutions de l'histoire de la musique. En effet, dès l'époque baroque la facture instrumentale évolue, les premiers orchestres se constituent, la pratique instrumentale individuelle est favorisée par la diffusion massive en France, en Italie, en Angleterre et dans les pays germaniques, de partitions, de recueils, de tablatures (Massip, 2007, p. 50). On peut par ailleurs résumer le XIX ${ }^{e}$ siècle $\mathrm{au}$ " triomphe de la musique instrumentale " selon Denizeau (2005, p. 170) avec la constitution de l'orchestre symphonique moderne, un nouveau développement de la facture instrumentale (et de la virtuosité), de nouveaux lieux pour faire et entendre de la musique (salons, salle de concert parfois de grandes dimensions (Michels, p. 435). Hoffmann l'un des premiers à parler de musique romantique déclarait : « Lorsqu'on parle de musique comme d'un art autonome, on ne devrait jamais penser qu'à la musique instrumentale [...] Elle est le plus romantique de tous les arts » (compte rendu de la $\mathrm{V}^{\mathrm{e}}$ symphonie de Beethoven in Allgemeine musikalische Zeitung, juillet 1810, cité par Michels, p. 437). Cette conception de la musique rejoint les vues de l'idéalisme allemand pour lequel l'art prend la place de la religion. Sans pouvoir démontrer le lien, il est à noter que dans les choix qui sont faits par les enseignants, la musique à programme et le poème symphonique sont diffusés en lieu et place, pourrait-on dire, de la musique religieuse. 
Figure 2. Fréquence de citation des cultures musicales traditionnelles diffusées, après regroupements par grands blocs continentaux

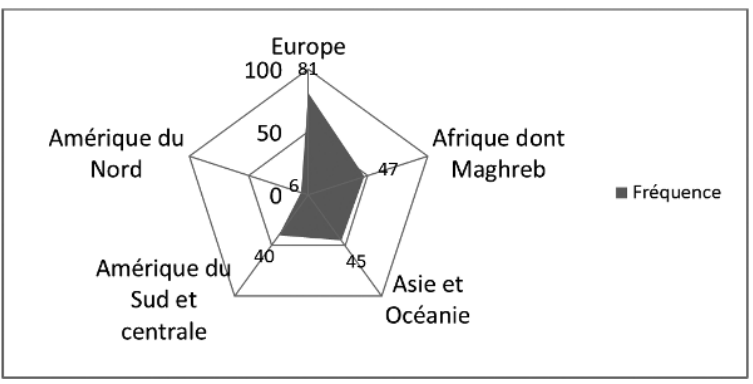

\section{Cultures musicales traditionnelles d'abord européennes}

Dans la catégorie des cultures musicales traditionnelles, c'est la diffusion du répertoire européen (français, 19\%, irlandais, 17\%, d'Europe de l'est, 15\%, espagnol, $10 \%$, allemand, $5 \%$, italien) qui prédomine sur les trois « blocs» Afrique, Asie-Océanie et Amérique du Sud. Ce sont les cultures musicales les plus proches des élèves, géographiquement, qui sont en jeu.

\section{Des genres musicaux caractéristiques des époques et des évolutions}

Plus précisément, ces premiers résultats généraux, référés aux travaux musicologiques généralistes (Chailley, 1958, Massin, 1985, Michels, 1990, Weidt, 1992, Denizeau, 1997, 2005, 2010) témoignent du fait que les enseignants semblent choisir principalement (cf. tableau 3), les genres musicaux parmi les plus représentatifs de chaque période, sinon ceux qui ont été les plus didactisés par les travaux en question : les ouvrages cités opèrent en quelque sorte une forme de prélèvement que l'on peut qualifier de transposition didactique, des savoirs produits par la recherche musicologique. Il en va ainsi du grégorien, des danses et des chansons des trouvères et des troubadours pour le Moyen Âge, ou encore, de la chanson polyphonique pour la Renaissance, de l'opéra et du concerto grosso pour le Baroque, de l'opéra buffa, comique et séria ou encore du concerto et de la symphonie pour la période classique, de la symphonie et de la musique à programme pour la période romantique, de l'électroacoustique et de la musique répétitive et concrète pour le $\mathrm{XX}$ e savant, $\mathrm{du}$ spiritual, du gospel, du blues, du jazz d'avant-guerre, de la comédie musicale et du rock pour le $\mathrm{XX}^{\mathrm{e}}$ populaire et jazz.

On peut y voir également un choix fondé sur l'ordre d'apparition des différents genres d'une époque à l'autre : c'est le cas, par exemple, pour la chanson polyphonique qui certes débute dans le bas-Moyen Âge (Ferrand 1999) mais dont le plein épanouissement se fait à la Renaissance, ou, encore, de l'opéra à l'époque baroque, de la musique à programme et du poème symphonique au XIX ${ }^{\mathrm{e}}$, de l'électroacoustique au $\mathrm{XX}^{\mathrm{e}}$. Toutefois on remarque une exception de taille à cette explication : l'atonalité, le dodécaphonisme, le sérialisme, courants représentatifs des nouveautés du $\mathrm{XX}^{\mathrm{e}}$ siècle sont très peu choisis par les enseignants, en raison disent-ils des difficultés liées à l'analyse de ce type d'œuvres.

On peut faire l'hypothèse que les évolutions de l'histoire de la musique expliquent implicitement les choix des enseignants d'autant que ces enseignants ont majoritairement suivi un cursus en musicologie. Cette hypothèse ne pourra être confirmée ou infirmée que dans une étude ultérieure. Ces grands équilibres, présents dans leurs réponses, peuvent aussi s'expliquer au regard de leurs pratiques vocales, instrumentales et d'écoutes sur l'ensemble de leur cursus (tableau 1). Pour autant, ces premières tentatives d'explications ne prouvent pas que la logique qui préside aux choix de l'enseignant soit celle-ci. Une des manières de vérifier l'acceptabilité de cette hypothèse est d'examiner les critères de choix des enseignants.

\section{Deuxième discussion}

Ces choix en partie explicites (genres et compositeurs cochés) et en partie implicites (grands équilibres analysés) rappellent - pour les musiques savantes seulement - les contenus d'enseignement des instructions et programmes de collège mis en œuvre de 1938 à 1977 : ils prescrivaient ces genres musicaux, ces compositeurs, ces grandes catégories dans l'ordre chronologique (Tripier-Mondancin, 2008, 2010). Au-delà des musiques savantes, ces résultats réfèrent aux préconisations récentes des programmes de 2008. S'agirait-il d'une sorte de déférence implicite et visiblement partagée, à l'égard 
Odile Tripier Mondancin

d'œuvres qui s'avèreraient incontournables (Snyders, 2012, p. 155)?

Si on considère avec les cognitivistes qu'enseigner « c'est favoriser l'apprentissage de trois composantes d'une connaissance : son contenu, ses conditions d'utilisation et sa formalisation ». (Musial, Pradère, \& Tricot, 2012, p. 29), alors rien ne prouve que la notion de genre, ainsi que les grands équilibres que nous venons d'analyser, soient explicitement enseignés.

En revanche, même si ces grands équilibres restent à l'état implicite, soit non enseignés, ils font partie de l'environnement musical auquel l'élève se trouve confronté au sein même de l'institution : « L'état implicite [de la connaissance] pourra, quant à lui, servir de point d'appui à l'apprentissage des états explicites d'une connaissance. » (ibid. p. 29).

Une étude fondée sur des pratiques effectives permettrait d'envisager si ces équilibres et notions acquièrent un statut de savoir enseigné ou s'ils en restent au statut de notion para voire proto musicale : vont-ils de soi, sont-ils nécessairement verbalisés, explicités, participent-ils à la compréhension de la musique?

Enfin, ces premières analyses ont tendance à introduire des nuances dans l'étude de Coulangeon lorsqu'il signale par exemple, à propos du diplôme dont l'effet, écrit-il, est délicat à interpréter : « la musique savante n'appartenant pas au même titre que la littérature classique à l'univers de la culture scolaire, on conçoit mal en quoi peut consister le rôle de l'école $[\ldots] »$ (p. 24-25). Se dessine ici certainement un rôle, en filigrane.

\section{LOGIQUES DE CHOIX}

\section{Critères en faveur de notions musicales perceptibles}

La question des critères de choix des cuvres diffusées a généré 55 valeurs manquantes, probablement parce que le questionnaire était long. Si l'on ne tient pas compte de ces valeurs manquantes, une première analyse fondée sur des statistiques descriptives (tableau 5) montre que les deux-tiers des enseignants choisissent les œuvres qu'ils diffusent avant tout, en fonction des « notions ou composantes musicales présentes dans l'œuvre comme par ex. monodie, polyphonie, bourdon, timbre $»(\operatorname{rang} 1)$.
Le deuxième ensemble est constitué de « critères liés à la faisabilité [du point de vue du professeur] par l'élève, de l'analyse auditive » ( $2^{\mathrm{e}}$ et $3^{\mathrm{e}}$ rang), en lien avec « l'époque ou la période " $\left(2^{\mathrm{e}}, 3^{\mathrm{e}}, 4^{\mathrm{e}}\right)$ et avec le « genre musical» $\left(2^{\mathrm{e}}, 3^{\mathrm{e}}\right)$. Si le premier critère fait état de notions musicales inhérentes aux compositions, le deuxième ensemble de critères réfère d'abord aux questions de capacité de traitement et de perception de l'information auditive, au sens de prise de conscience par les élèves, puis complète le premier critère par des connaissances historico-catégorico-techniques du fait musical.

Le $3^{\text {e }}$ bloc de critères (rangs 4 à 8 ) prolonge le précédent mais fait apparaitre des réponses plus «éclatées »: « l'époque », " la faisabilité de l'analyse auditive », « le genre », « la composition » et «le compositeur », retiennent l'attention des enseignants. Les préférences des enseignants influenceraient peu leurs choix. Le $4^{\mathrm{e}}$ bloc (rangs 8 à 11 ) est constitué de critères liés à la sensibilité ( «émotions et sentiments qui peuvent être suscités par telle ou telle ouvre »), aux " programmes de français », aux « découvertes récentes du professeur ». La question du « chef-d'œuvre » (ou du statut de l'œuvre) (rang 12 à 13) ne semble pas du tout préoccuper les enseignants (Snyders, [1999-2000], 2012).

Les réponses à la question ouverte (Autre(s) critère(s) de choix des genres musicaux ?) montrent que les œuvres sont très rarement choisies en fonction, « d'un lien» avec le chant étudié durant la " séquence » (3 occurrences) soit, « avec le projet musical réalisé par la classe » (MEN, 2008), ou encore en fonction des « compétences recherchées » (1 occurrence). Les concepts nouvellement parus dans les programmes d'août 2008 (projet musical, compétences) ne font l'objet que de peu d'occurrences (5).

À première vue, ce ne sont donc pas le genre musical et le compositeur ou encore la transmission de la notion de chef-d'œuvre qui guident en premier lieu le choix des enseignants. Semble-t-il, le choix des œuvres s'opère dans une combinaison complexe d'un ensemble de critères.

\section{Effet de primauté mais pas de récence}

Si l'effet de récence sur les derniers éléments de la liste ne s'observe pas (tableau 5), en revanche, les résultats donnent à penser qu'un effet de primauté 


\begin{tabular}{|c|c|c|c|c|c|}
\hline 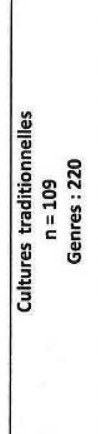 & & 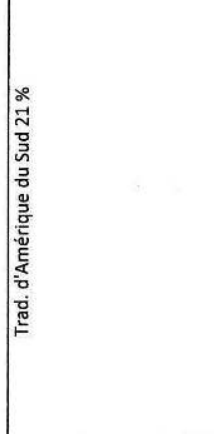 & 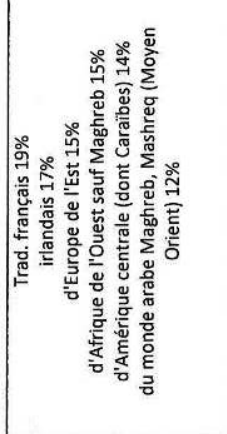 & 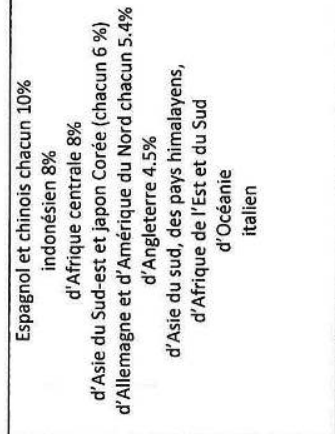 & 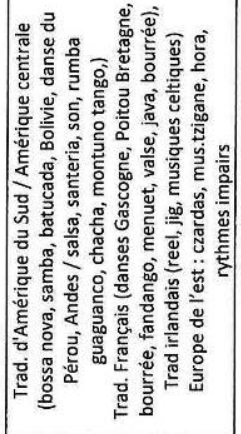 \\
\hline 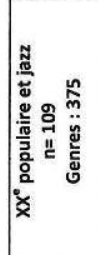 & & 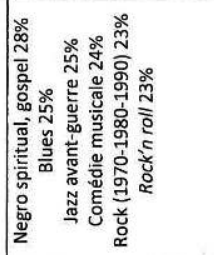 & 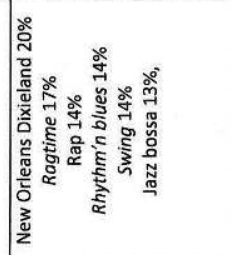 & 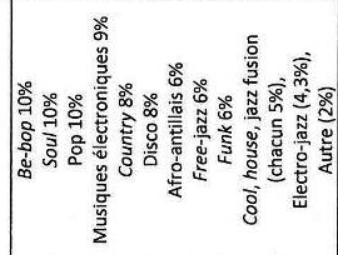 & 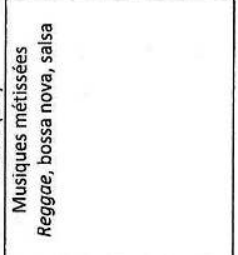 \\
\hline 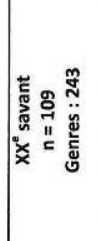 & & 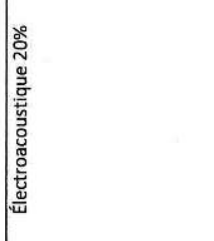 & 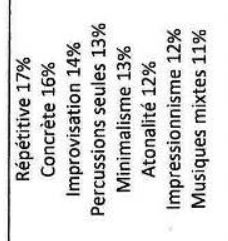 & 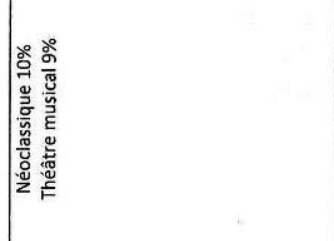 & 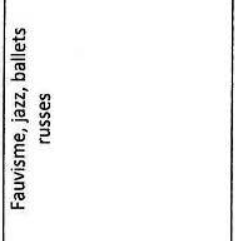 \\
\hline 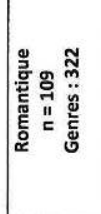 & & 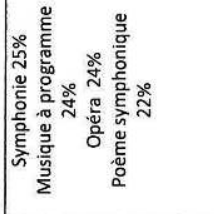 & 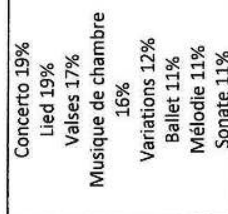 & 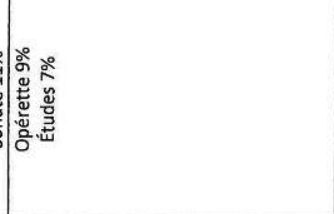 & 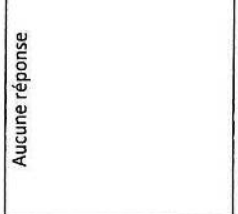 \\
\hline 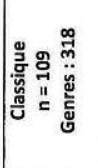 & & 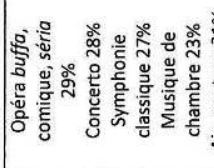 & 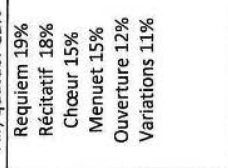 & & 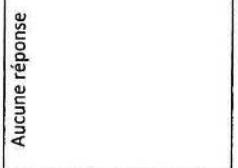 \\
\hline 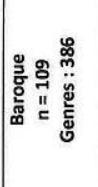 & 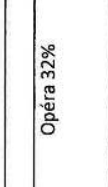 & 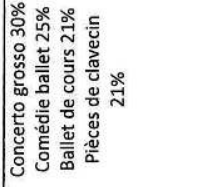 & 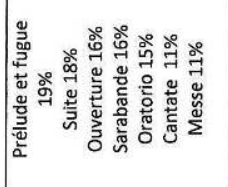 & & 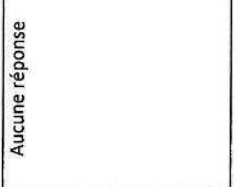 \\
\hline 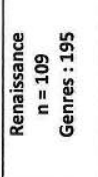 & 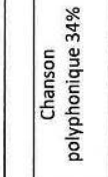 & 递 & & 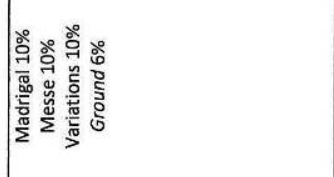 & 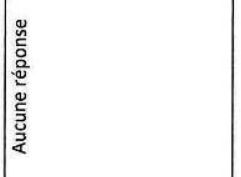 \\
\hline 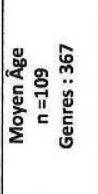 & 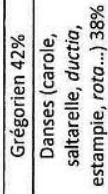 & 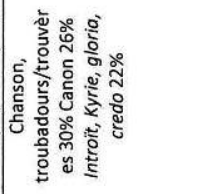 & 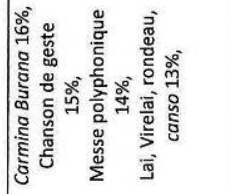 & 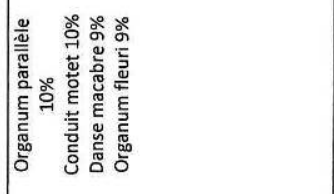 & 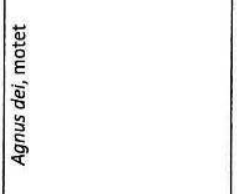 \\
\hline 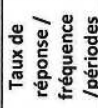 & 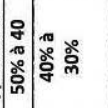 & 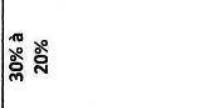 & ذై & 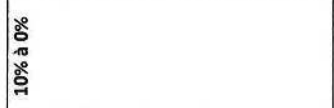 & 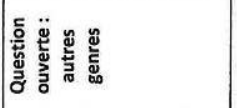 \\
\hline
\end{tabular}




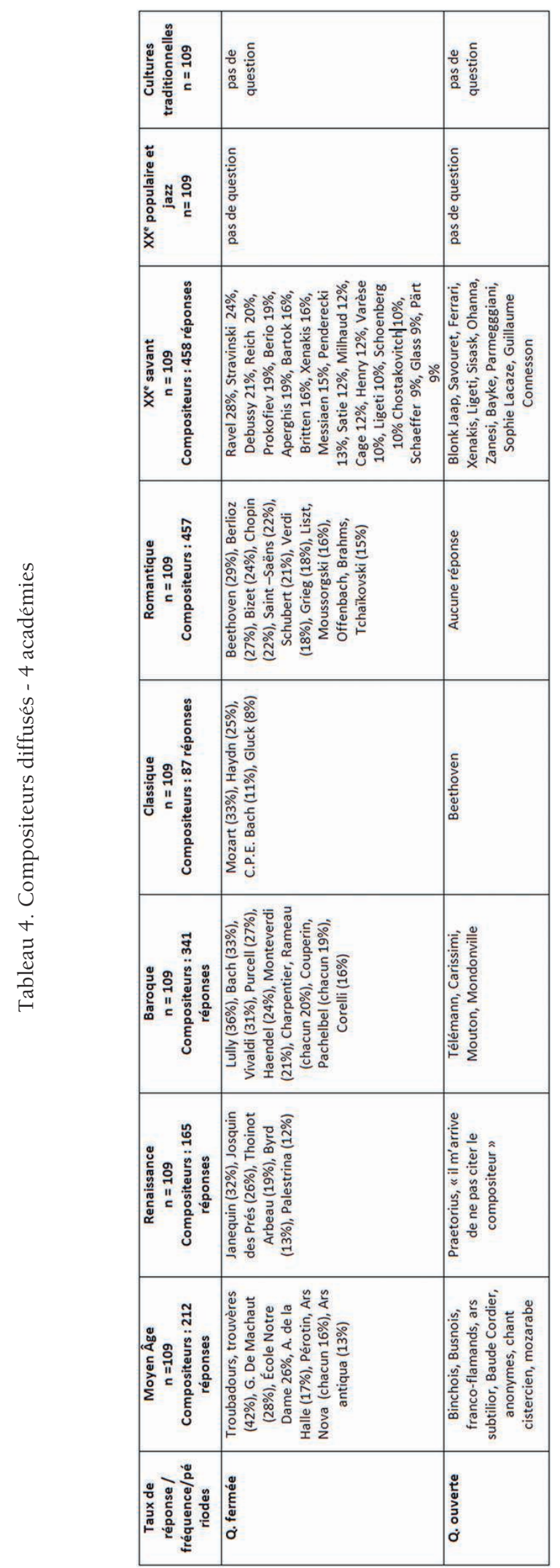


Odile Tripier Mondancin

opère (l'item 1 « notions musicales » est le plus retenu). Afin de nuancer la portée de cet effet, nous avons procédé à des tests statistiques sur les rangs pour parvenir au rang moyen.

Ce critère permet d'évaluer le potentiel d'une donnée à représenter l'échantillon auquel elle appartient. C'est la donnée qui a le plus petit rang moyen qui est la plus représentative des réponses. Or, si on s'attache au premier quartile de rang (3 à 5 premiers rangs) il s'avère que les deux critères ex aequo les plus représentatifs sont « les critères liés à la faisabilité technique de l'analyse » et «l'époque ou la période » à laquelle l'œuvre réfère (tableau 6). En $2^{\mathrm{e}}$ position, on trouve le critère du genre musical. Le programme d'histoire semble jouer un rôle (rangs 1 à 11).
Pour ce qui est des raisons énoncées lors des entretiens post-observation de classe dans l'enquête de 2005, elles témoignaient également (1) de l'importance des notions musicales dans les choix des enseignants, (2) d'une recherche de cohérence entre les différentes activités, puis (3) des goûts musicaux des enseignants. Parmi les notions musicales, on trouve en rang 1 de réponses sur 17 propositions, neuf propositions relatives aux notions-matériaux du musical : par exemple, découverte description et identification des cordes frottées, des paramètres du son, dont celui de la hauteur, de l'orchestre symphonique, des différents timbres de voix, structure d'un blues, structure d'une pièce I gondolieri, extraite de L'Italienne à Alger de Rossini, indices caractérisant le

Tableau 5. Critères de choix des œuvres diffusées analysées en classe d'éducation musicale au collège (distribution ou effectifs des réponses)

\begin{tabular}{|c|c|c|c|c|c|c|c|c|c|c|c|c|c|c|}
\hline \multicolumn{14}{|c|}{ Question : Lorsque vous choisissez les œuvres ou compositions à faire écouter et analyser à vos élèves, vous le faites plutôt en fonction : } & \multirow[b]{2}{*}{ TOTAL } \\
\hline Items & \begin{tabular}{|l|} 
Effectifs \\
rang 1
\end{tabular} & $\begin{array}{l}\text { Effectifs } \\
2\end{array}$ & \begin{tabular}{|l|} 
Effectifs \\
3
\end{tabular} & \begin{tabular}{|l|} 
Effectifs \\
4
\end{tabular} & $\begin{array}{l}\text { Effectifs } \\
5\end{array}$ & \begin{tabular}{|l|} 
Effectifs \\
6
\end{tabular} & \begin{tabular}{|l|} 
Effectifs \\
7
\end{tabular} & \begin{tabular}{|l|} 
Effectifs \\
8
\end{tabular} & \begin{tabular}{|l|} 
Effectifs \\
9
\end{tabular} & \begin{tabular}{|l|} 
Effectifs \\
10
\end{tabular} & \begin{tabular}{|l|} 
Effectifs \\
11
\end{tabular} & \begin{tabular}{|l|} 
Effectifs \\
12
\end{tabular} & \begin{tabular}{|l|} 
Effectifs \\
13
\end{tabular} & \\
\hline $\begin{array}{l}\text { des notions/composantes (ex. } \\
\text { monodie, bourdon...) }\end{array}$ & 35 & 5 & 2 & 5 & 2 & 1 & 0 & 0 & 2 & 0 & 1 & 1 & 0 & 54 \\
\hline $\begin{array}{l}\text { de la composition elle-même } \\
\text { (ex. le Printemps/Vivaldi) }\end{array}$ & 1 & 4 & 1 & 2 & 7 & 3 & 8 & 6 & 9 & 7 & 3 & 3 & 0 & 54 \\
\hline $\begin{array}{l}\text { de critères liés à la faisabilité } \\
\text { de l'analyse }\end{array}$ & 2 & 12 & 11 & 5 & 8 & 6 & 0 & 2 & 4 & 2 & 2 & 0 & 0 & 54 \\
\hline du genre musical & 1 & 8 & 14 & 6 & 5 & 10 & 1 & 7 & 1 & 0 & 1 & 0 & 0 & 54 \\
\hline $\begin{array}{l}\text { du compositeur/de la } \\
\text { compositrice/du courant/de } \\
\text { l'école }\end{array}$ & 1 & 3 & 1 & 6 & 6 & 6 & 7 & 10 & 6 & 4 & 3 & 1 & 0 & 54 \\
\hline de l'époque/de la période & 6 & 9 & 9 & 14 & 6 & 2 & 4 & 2 & 0 & 2 & 0 & 0 & 0 & 54 \\
\hline de vos préférences musicales & 2 & 4 & 3 & 2 & 2 & 6 & 6 & 8 & 3 & 6 & 6 & 3 & 3 & 54 \\
\hline de vos découvertes récentes & 0 & 1 & 2 & 2 & 2 & 4 & 3 & 6 & 8 & 7 & 13 & 6 & 0 & 54 \\
\hline $\begin{array}{l}\text { des émotions et sentiments } \\
\text { suscités par l'œuvre }\end{array}$ & 3 & 3 & 4 & 5 & 4 & 3 & 8 & 2 & 12 & 4 & 4 & 2 & 0 & 54 \\
\hline du programme d'histoire & 3 & 3 & 5 & 5 & 7 & 7 & 5 & 3 & 3 & 8 & 3 & 2 & 0 & 54 \\
\hline du programme de français & 0 & 0 & 0 & 2 & 2 & 5 & 6 & 6 & 2 & 10 & 9 & 10 & 2 & 54 \\
\hline $\begin{array}{l}\text { de ce que vous considérez } \\
\text { comme un chef-d'œuvre }\end{array}$ & 0 & 1 & 2 & 0 & 3 & 1 & 5 & 2 & 4 & 4 & 7 & 23 & 2 & 54 \\
\hline d'un autre(s) critère(s) & 0 & 1 & 0 & 0 & 0 & 0 & 1 & 0 & 0 & 0 & 2 & 3 & 47 & 54 \\
\hline Total & 54 & 54 & 54 & 54 & 54 & 54 & 54 & 54 & 54 & 54 & 54 & 54 & 54 & \\
\hline
\end{tabular}


Odile Tripier Mondancin

style jazz, pulsé/non pulsé. Il y aurait donc concordance entre ces résultats.

Cette nouvelle analyse permet de conclure que si (1) les notions ou composantes musicales inhérentes à l'œuvre jouent un rôle prépondérant dans le choix des œuvres à écouter en classe, il n'en demeure pas moins que les critères relatifs (2) à la faisabilité de l'analyse (ce qui correspond aux questions de perception auditive), (3) à la période dans laquelle l'œuvre est composée, (4) aux questions de genre musical et enfin (5) au programme d'histoire, ne sont pas négligeables. ou période, (2) inter-esthétiques, (3) toutes esthétiques confondues.

\section{Notions musicales spécifiques ou transversales}

Quelle que soit l'époque ou l'esthétique, deux types de raisons sont prédominants: les notions véhiculées par les œuvres, en lien avec une époque (87 propositions) ainsi que la volonté « d'illustrer, de faire des parallèles avec le cours d'histoire, pour donner du sens $»(55)$. C'est l'histoire qui est invo-

\begin{tabular}{|c|c|c|c|}
\hline $\begin{array}{l}\text { Lorsque vous choisissez les ouvres ou compositions à faire écouter et } \\
\text { analyser à vos élèves, vous le faites plutôt en fonction : }\end{array}$ & $\operatorname{Rg} 1$ à 3 & $\operatorname{Rg} 1$ à 5 & $\operatorname{Rg} 1$ à 11 \\
\hline des notions ou composantes & 4,00 & 4,40 & 6,09 \\
\hline de la composition elle-même & 6,00 & 4,80 & 4,18 \\
\hline de critères techniques/faisabilité de l'analyse & 2,33 & 2,20 & 4,18 \\
\hline du genre musical & 3,00 & 3,00 & 4,45 \\
\hline du compositeur/compositrice/courant, école & 6,33 & 4,80 & 4,09 \\
\hline de l'époque/période & 2,33 & 2,20 & 4,82 \\
\hline de vos préférences musicales & 5,00 & 5,20 & 4,36 \\
\hline de vos découvertes récentes & 6,67 & 6,20 & 4,82 \\
\hline des émotions et sentiments suscités par l'œuvre & 4,67 & 4,40 & 4,18 \\
\hline du programme d'histoire & 4,33 & 3,60 & 3,91 \\
\hline du programme de français & 7,67 & 6,80 & 5,00 \\
\hline de ce que vous considérez comme chef-d'œuvre & 6,67 & 6,20 & 5,64 \\
\hline Autre & 7,33 & 7,00 & 7,36 \\
\hline
\end{tabular}

Autres logiques de choix : raisons pour lesquelles les enseignants ont choisi d'aborder ces genres musicaux

L'analyse précédente peut être complétée grâce aux réponses à la question ouverte sur les raisons pour lesquelles les enseignants choisissent à chaque époque, tel genre plutôt qu'un autre.

D'une manière quantitative, un peu moins de la moitié des enseignants a répondu à cette question ouverte. Les genres musicaux classiques $\left(\mathrm{XVIII}^{\mathrm{e}}\right)$ sont ceux qui suscitent le moins de raisons par enseignants (entre une et trois), tandis que les genres baroques, ceux de la Renaissance et ceux de la période romantique génèrent entre une et sept raisons. Trois types d'analyse qualitative/quantitative des catégories de raisons peuvent être faits (1) au sein d'une esthétique quée le plus souvent quand ce n'est pas dans l'ordre décroissant, l'histoire des arts, le français, les arts plastiques, l'EPS. L'époque baroque semble davantage propice aux approches interdisciplinaires : littérature, sciences, arts de l'espace (jardins), peintures. Ces réponses ont tendance à corroborer l'analyse par rang de moyenne. La référence à l'histoire des arts semble liée au fait que l'enquête se déroule en 2010, soit deux ans après la parution d'un texte sur l'organisation de l'histoire des arts (MEN 2008).

Le détail des notions à enseigner montre que, soit, elles font consensus et sont transversales tout en fonctionnant par oppositions (comme monodie/ polyphonie, sacré/profane pour les genres du Moyen Âge), soit, elles ne sont citées qu'en très petit nombre. Il en va ainsi des notions de temps pulsél non pulsé et de sonorités spécifiques, des notions baroques d'écriture contrapuntique, d'ornementa- 
tion, de basse continue, de basse obstinée, d'écriture en trio, de figuralismes, de musique concertante, de facture instrumentale, d'écriture fuguée, de choral, de forme rondo, des notions classiques de forme sonate, de thème, de cadence, de respiration, de pluralité de formations, des notions romantiques de virtuosité, de forme lied, de musique de salon, d'orchestration, des notions du XIX ${ }^{\mathrm{e}}$ et du XXI ${ }^{\mathrm{e}}$ siècles liées aux nouvelles technologies de travail sur le son, enfin, d'instrumentation caractéristique de genres spécifiques aux musiques traditionnelles.

Toutes les esthétiques sont concernées par la question des notions hormis les genres populaires $\mathrm{du} \mathrm{XX}^{\mathrm{e}}$ et le jazz : c'est pour leur lien avec l'histoire et la société (5) mais c'est aussi pour la proximité (ou l'éloignement dans certains cas) et la contemporanéité de ces genres musicaux avec les élèves (5) qu'ils sont choisis.

\section{Rapport aux ouvres : appréciation, préférences, accessibilité de l'ouvre}

Le troisième type de raisons émergentes concerne la question du rapport personnel aux ouvres en termes de plaisir et d'appréciation : le fait que tel genre "plaît aux élèves », ou que " c'est agréable à écouter »ou " par affinité par passion, par goût personnel, par préférence » (15). Inversement, très rarement quelques enseignants justifient parfois le fait de ne pas aborder tel genre parce qu'il est jugé " insipide» (en référence à des genres du XVIII ${ }^{\mathrm{e}}$ ). Un dernier signale que le Moyen Âge n'est pas « du tout dans ses préférences musicales », pour autant, il est « intéressé par la musique de danses et le chant grégorien » dans leur rapport à des films (Le nom de la rose, Sacré Graal).

Le quatrième type de raisons porte sur la supposée accessibilité de l'œuvre : « l'appropriation, l'ana- lyse des genres musicaux du Moyen Âge est assez facile, aisée (structures simples) type de raisons est donné également à propos des cultures musicales traditionnelles (5) et des genres baroques (3).

\section{Ouverture à d'autres langages musicaux}

Une catégorie de raisons est énoncée quasi exclusivement à propos des genres traditionnels : «l'ouverture culturelle, la confrontation à d'autres langages musicaux », " toute musique vaut la peine d'être écoutée, d'où qu'elle vienne » (9).

\section{Des réponses qui se corroborent par triangulation}

Ces résultats corroborent et complètent les réponses à la question fermée en éventail. En revanche, ils confirment que la centration des enseignants sur le désir, les besoins, la motivation des élèves qui conduit l'enseignant à faire des choix correspondants à ce qui est déjà apprécié, centration relevée par Mialaret, ne se vérifie que dans une moindre mesure les déclarations de ces enseignants (figure 3).

\section{Troisième discussion}

Le lien entre critères de choix déclarés et savoirs enseignés ne va pas de soi. Pour autant certains critères correspondent à des objets musicaux au sens chevallardien qui peuvent être des candidats pour être enseignés. Même si nous n'avons pas la preuve qu'ils le soient, il y a de fortes chances que ce soit le

Figure 3. Modélisation des critères de choix d'une œuvre diffusée (à lire de gauche à droite)

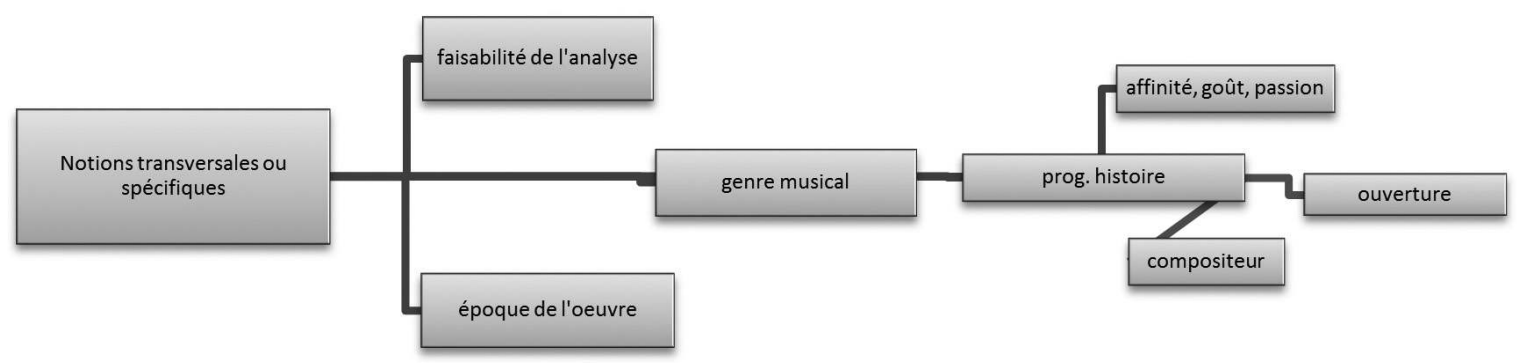


cas. Dans tous les cas, même si nous ne pouvons pas la confirmer, l'hypothèse peut être posée.

Dans la liste de critères que nous avions proposée, il en va ainsi d'un premier ensemble qui correspond (dans l'ordre décroissant) aux notions ou composantes de l'œuvre, à la notion d'époque, de genre musical, de composition (titre de l'œuvre), de compositeur ou encore des savoirs que les programmes d'histoire et de français infèrent, enfin, de chef-d'œuvre. Ces critères peuvent être regroupés sous le concept de candidats pour devenir des savoirs enseignables/enseignés. La question du statut du compositeur et de la composition n'est toujours pas résolue à ce stade : l'œuvre est-elle dépersonnalisée dès lors qu'elle est enseignée, son titre est-il donné même si ce n'est qu'en tant que renseignement? Il semble tout de même que ce ne soit pas la priorité (cf. rangs de moyenne).

Un deuxième ensemble de critères serait susceptible de constituer un réservoir de savoir-faire enseignés étroitement intriqués avec les précédents: critères en lien avec la faisabilité de l'analyse auditive soit avec les capacités de traitement auditif.

Quant aux préférences musicales et aux découvertes récentes de l'enseignant, elles seraient relatives au domaine des connaissances et expériences de l'enseignant, tandis que les émotions suscitées seraient du domaine des expériences subjectives des élèves et dès lors de leurs connaissances, de leurs représentations, verbalisées ou non dans la classe. On sait que les émotions ont un effet sur l'engagement des apprenants dans la situation d'apprentissage et non pas directement sur les processus. Elles permettent de mobiliser des ressources cognitives qui vont être utilisées pour mettre en œuvre les processus d'apprentissage (Damasio, 1999). Elles ne peuvent donc pas être candidates en tant que savoirs à enseigner mais peuvent être mobilisées à leur service.
Néanmoins, chaque critère est susceptible de basculer dans une autre catégorie, suivant le statut qui lui est accordé dans la classe.

Les deux critères principaux de choix témoignent a priori malgré tout d'une importance accordée à l'enseignement de connaissances déclaratives spécifiques aux œuvres (notions, époque, genre, compositeur, programme d'histoire) mais aussi procédurale (méthode et savoir - faire, faisabilité de l'analyse auditive). Les émotions et sentiments restent en marge de cette activité d'écoute.

\section{VARIABLES EXPLICATIVES DE CES CHOIX}

Au-delà des raisons invoquées par les enseignants, ou des hypothèses que l'on peut faire de liens avec les évolutions musicales, d'autres variables peuvent expliquer leurs choix. Afin de vérifier ou d'infirmer notre hypothèse initiale (influence réciproque entre les quatre variables indépendantes et les choix des enseignants), nous avons appliqué des analyses de variance systématisées à l'ensemble de nos données. L'analyse de variance permet de mettre en évidence si les distributions (scores) sont significativement différentes pour chacune de ces variables indépendantes: « On compare les moyennes à l'intérieur et/ou entre les échantillons. L'anova concerne le test d'hypothèses sur des moyennes » (Kinnear, Gray, 2005, p. 199. Nous les avons assorties du test de Tukey pour les variables ayant au moins trois modalités (l'académie, les années d'ancienneté).

En ce qui concerne les critères de choix des œuvres, seules deux variables sur les quatre sont en relation de manière significative : le genre (homme/ femme) et l'académie d'appartenance.

En ce qui concerne la diffusion de tel genre ou tel compositeur, c'est l'ancienneté et l'académie d'appartenance qui induiraient significativement une variance.

Tableau 7. Catégorisation des critères et raisons selon les catégories de savoir (cf. cadre théorique) et selon leur caractère

\begin{tabular}{|l|l|l|l|}
\hline Savoirs enseignables/enseignés & $\begin{array}{l}\text { Savoir-faire enseignables/ } \\
\text { enseignés }\end{array}$ & $\begin{array}{l}\text { Connaissances, expériences } \\
\text { personnelles }\end{array}$ & $\begin{array}{l}\text { Informations communiquées/ } \\
\text { non communiquées }\end{array}$ \\
\hline $\begin{array}{l}\text { Notions musicales, époque, } \\
\text { genre, composition, compo- } \\
\text { siteur, notions transversales } \\
\text { d'histoire, de français, en lien } \\
\text { avec autres arts }\end{array}$ & $\begin{array}{l}\text { Analyse auditive, faisabilitéé } \\
\text { du traitement }\end{array}$ & $\begin{array}{l}\text { Préférences, découvertes, } \\
\text { émotions sentiments susci- } \\
\text { tés, rapport à l'oeuvre }\end{array}$ & $\begin{array}{l}\text { Compositeur, } \\
\text { Titre de l'œuvre }\end{array}$ \\
\hline
\end{tabular}


Le genre et l'académie d'appartenance expliquent certains critères de choix

Le genre (homme/femme) est en lien très significativement avec certains critères de choix comme la faisabilité de la perception/analyse auditive $(\mathrm{p}<0,04)$ et significativement avec l'époque $(\mathrm{p}<, 011)$, les émotions et sentiments qui peuvent être suscités par telle ou telle œuvre ( $\mathrm{p}<, 067)$. Quant à l'académie d'appartenance, elle ne serait en relation qu'avec le critère des émotions et sentiments suscités par l'œuvre (plus significatif à Toulouse qu'à Bordeaux : $\mathrm{p}<, 013$ ).

\section{Ancienneté en lien avec le choix de certains genres et compositeurs}

L'ancienneté dans le métier des enseignants (tableau 8) est en relation avec le choix de tel ou tel genre musical tel ou tel compositeur. Plus précisément, les enseignants qui ont entre 16 ans et 20 ans d'ancienneté proposent plus spécifiquement certains genres et compositeurs que les enseignants moins expérimentés en nombre d'années ( 6 à 10 ans) : ceux du XVII ${ }^{\mathrm{e}}$ (chaconne), du XVIII ${ }^{\mathrm{e}}$ (Air), du XIX ${ }^{\mathrm{e}}$ (poème symphonique, musique de chambre, Berlioz, Bizet), du XXe (Ravel, Xenakis, Varèse, comédie musicale, blues), certains traditionnels français.

\section{Académie d'appartenance en lien avec des genres ou des compositeurs}

L'influence réciproque entre l'académie et le répertoire choisi s'observerait particulièrement lorsque les enseignants sont en poste à Bordeaux plus qu'à Toulouse ou Limoges, toutes esthétiques confondues du Moyen Âge, aux musiques traditionnelles d'Europe de l'Est, espagnoles et afro-antillaises (Toulouse, Limoges).

Quasiment pas de lien entre genre, type de collège et répertoires choisis

Le type de collège (ZEP/non ZEP) dans lequel les répondants enseignent n'aurait pas de lien significatif avec le choix d'un genre musical ou d'un compositeur hormis sur quelques genres choisis appartenant à la Renaissance (Diférencias $\mathrm{p}<, 005$, la frottola $\mathrm{p}<, 005$ ).

Enfin, le fait d'être un enseignant homme ou femme ne semble pas lié d'après les réponses de cet échantillon, au choix de tel ou tel répertoire, sinon à des répertoires très peu choisis (Léonin, l'Ars nova, la synthèse sonore, le disco, les traditionnels d'Amérique du Nord).

Tableau 8. Test de Tukey reposant sur la variance des genres et compositeurs, selon l'ancienneté dans le métier

\begin{tabular}{|c|c|c|}
\hline Genres et compositeurs choisis & $\begin{array}{l}\text { Rappel du taux de fré- } \\
\text { quence de ces choix }\end{array}$ & Analyse de variance par ancienneté \\
\hline Chaconne & $9.09 \%$ & + de 21 à 25 ans que de 06 à 10 ans $(\mathrm{p}<, 015)$ \\
\hline Air & $20.91 \%$ & $\begin{array}{l}\text { + de } 16 \text { à } 20 \text { ans que de } 1 \text { à } 5 \text { ans }(p<, 002) \text {, que de } \\
6 \text { à } 10 \text { ans }(p<0,003) \text {, que de } 21 \text { à } 25(p<, 041) \text {, que } \\
\text { de } 26 \text { à } 36 \text { ans }(p<, 017)\end{array}$ \\
\hline $\begin{array}{l}\text { Poème symphonique } \\
\text { Musique de chambre (dont pour piano) }\end{array}$ & $\begin{array}{l}22.73 \% \\
16.36 \%\end{array}$ & $\begin{array}{l}\text { + de } 16 \text { à } 20 \text { ans que de } 6 \text { à } 10 \text { ans }(\mathrm{p}<, 032) \\
+ \text { de } 16 \text { à } 20 \text { ans que de } 6 \text { à } 10 \text { ans }(\mathrm{p}<, 035)\end{array}$ \\
\hline $\begin{array}{l}\text { Berlioz H. (1803-1869) } \\
\text { Bizet G. (1838-1875) }\end{array}$ & $\begin{array}{l}27.27 \% \\
24,55 \% \\
\end{array}$ & $\begin{array}{l}\text { + de } 16 \text { à } 20 \text { ans que de } 6 \text { à } 10 \text { ans }(\mathrm{p}<, 039) \\
+ \text { de } 16 \text { à } 20 \text { ans que de } 6 \text { à } 10 \text { ans }(\mathrm{p}<, 037)\end{array}$ \\
\hline $\begin{array}{l}\text { Ravel M. (1875-1937) } \\
\text { Xenakis I. (1922-2001) } \\
\text { Varèse E. (1883-1965) }\end{array}$ & $\begin{array}{l}28.18 \% \\
16.36 \% \\
10.91 \%\end{array}$ & $\begin{array}{l}\text { + de } 16 \text { à } 20 \text { ans que de } 6 \text { à } 10 \text { ans }(\mathrm{p}<, 038) \\
+ \text { de } 16 \text { à } 20 \text { ans que de } 6 \text { à } 10 \text { ans }(\mathrm{p}<, 034) \\
+ \text { de } 21 \text { à } 25 \text { ans que de } 6 \text { à } 10 \text { ans }(\mathrm{p}<, 042)\end{array}$ \\
\hline $\begin{array}{l}\text { Comédie musicale } \\
\text { Blues }\end{array}$ & $\begin{array}{l}9.09 \% \\
8.18 \% \\
\end{array}$ & $\begin{array}{l}\text { + de } 16 \text { à } 20 \text { ans que de } 6 \text { à } 10 \text { ans }(\mathrm{p}<, 028) \\
+ \text { de } 16 \text { à } 20 \text { ans que de } 6 \text { à } 10 \text { ans }(\mathrm{p}<, 035)\end{array}$ \\
\hline Traditionnels français & $19.09 \%$ & + de 16 à 20 ans que de 6 à 10 ans $(\mathrm{p}<, 015)$ \\
\hline
\end{tabular}




\section{Quatrième discussion}

Ces résultats sembleraient aller à l'encontre des tendances d'écoute des françaises et des français selon l'enquête de 1997 analysée par Coulangeon (2003, p. 64) qui fait ressortir une partition sexuée des goûts musicaux. En effet, selon les résultats des analyses de variance, les enseignants d'éducation musicale ne favoriseraient en rien, ou en d'autres termes, ne reproduiraient pas la partition entre les femmes qui écouteraient/préfèreraient de manière plus prononcée les variétés que les hommes.

Si le genre et le type de collège n'induisent pas de variance dans les choix, on peut conclure que l'enseignement proposé au moins sur les quatre académies questionnées est relativement homogène. Cela dit, il resterait à déterminer en quoi consiste plus exactement l'effet « académie » relevé : seraitce le poids des contenus proposés dans la formation continue, les recommandations d'IA-IPR (Inspecteurs Pédagogiques Régionaux), les échanges inter-profes- seurs, l'offre culturelle qui est en jeu ? Si comme nous l'avons dit dans le cadre théorique, le professeur se substitue au travail de la noosphère pour choisir les compositeurs et les genres qu'il déclare faire écouter, il semble qu'il ne soit pas seul face à ces choix. Il n'est pas seul à travailler à la transposition didactique. Mais la présente étude ne permet pas d'aller plus loin dans cette analyse.

\section{CONCLUSION : VERS UN « RÉPERTOIRE SCOLAIRE " CHOISI POUR SES VERTUS DIDACTIQUES}

Le réel est inépuisable, l'approche par questionnaire et l'outil statistique ont leurs limites dans l'objectivation et l'explicitation de ce réel. Parmi ces limites, on peut relever par exemple, les propositions qui ont été faites dans les questions en éventail. Le fait de donner à cocher une liste de genres musicaux issus d'études universitaires de la musicologie histo-

Tableau 9. Test de Tukey reposant sur l'analyse de variance des genres et compositeurs, selon l'académie

\begin{tabular}{|c|c|c|}
\hline Genres et compositeurs choisis & $\begin{array}{l}\text { Rappel du taux de fré- } \\
\text { quence de ces choix }\end{array}$ & Analyse de variance par académie \\
\hline $\begin{array}{l}\text { Chanson polyphonique } \\
\text { Chanson de geste } \\
\text { Lai, virelai, rondeau, canso } \\
\text { De la Halle A. (1240-1287) }\end{array}$ & $\begin{array}{l}30 \% \\
15.45 \% \\
13.64 \% \\
17.27 \%\end{array}$ & $\begin{array}{l}\text { + à Bordeaux qu'à Toulouse }(\mathrm{p}<, 038) \\
+ \text { à Bordeaux qu'à Limoges }(\mathrm{p}<, 024) \text { et qu'à Toulouse } \\
(\mathrm{p}<, 026) \\
+ \text { à Bordeaux qu'à Limoges }(\mathrm{p}<, 032) \text { et qu'à Toulouse } \\
(\mathrm{p}<, 015) \\
+ \text { à Bordeaux qu'à Limoges }(=, 022) \text { et qu'à Toulouse } \\
(\mathrm{p}<, 000)\end{array}$ \\
\hline $\begin{array}{l}\text { Madrigal } \\
\text { Messe }\end{array}$ & $\begin{array}{l}10.00 \% \\
10.00 \% \\
\end{array}$ & $\begin{array}{l}\text { + à Bordeaux qu'à Toulouse }(\mathrm{p}<, 023) \\
+ \text { à Bordeaux qu'à Toulouse }(\mathrm{p}<, 029)\end{array}$ \\
\hline $\begin{array}{l}\text { Variations } \\
\text { Toccata } \\
\text { Chaconne } \\
\text { Passacaille } \\
\text { Corelli A. (1653-1713) } \\
\text { Marin Marais (1656-1728) }\end{array}$ & $\begin{array}{l}11.82 \% \\
10.00 \% \\
9.09 \% \\
8.18 \% \\
16.36 \% \\
15.45 \%\end{array}$ & $\begin{array}{l}\text { + à Bordeaux qu'à Toulouse }(\mathrm{p}<, 021) \\
+ \text { à Bordeaux qu'à Limoges }(\mathrm{p}<, 027) \text { et qu'à Toulouse } \\
(\mathrm{p}<, 003) \\
\text { + à Clermont qu'à Toulouse }(\mathrm{p}<, 047) \\
+ \text { à Bordeaux qu'à Toulouse }(\mathrm{p}<, 028) \\
\text { + à Bordeaux qu'à Toulouse }(\mathrm{p}<, 025) \\
\text { + à Bordeaux qu'à Toulouse }(\mathrm{p}<, 031)\end{array}$ \\
\hline Mahler G. (1860-1911) & $12.73 \%$ & + à Bordeaux qu'à Toulouse $(\mathrm{p}<, 001)$ \\
\hline $\begin{array}{l}\text { Musique concrète } \\
\text { Varèse E. (1883-1965) }\end{array}$ & $\begin{array}{l}16,36 \% \\
10,91 \%\end{array}$ & $\begin{array}{l}\text { + à Bordeaux qu'à Limoges }(\mathrm{p}<, 022) \text { et qu'à Toulouse } \\
(\mathrm{p}<, 013) \\
+ \text { à Bordeaux qu'à Toulouse }(\mathrm{p}<, 035)\end{array}$ \\
\hline Afro-antillais & $8.18 \%$ & + à Bordeaux qu’à Toulouse $(\mathrm{p}<, 015)$ \\
\hline Trad. d'Europe de l'Est & $11.82 \%$ & + à Limoges qu'à Toulouse $(\mathrm{p}<, 040)$ \\
\hline Traditionnel espagnol & $10.91 \%$ & $\begin{array}{l}\text { + à Bordeaux qu'à Clermont }(\mathrm{p}<, 018) \text { et qu'à Toulouse } \\
(\mathrm{p}<, 000) \text { et } \\
\text { + à Limoges qu'à Toulouse }(\mathrm{p}<, 020)\end{array}$ \\
\hline
\end{tabular}


rique n'entretient-il pas l'illusion qu'il faudrait qu'il y ait « conformité » (Chevallard, p. 16) entre les savoirs enseignés et les savoirs savants? Sans doute vaudrait-il mieux partir des observations de situations de classe mais dans ce cas les études menées ne reflèteraient pas les grandes tendances que notre étude avait pour objectif d'approcher. En outre, la volonté de produire des données quantitatives et dès lors d'utiliser des nomenclatures générales (genre, compositeur) n'autorisent pas une segmentation fine des œuvres écoutées en classe, tout comme l'enquête sur les goûts musicaux des français ne permet pas de nuancer les préférences musicales en particulier des classes moyennes et populaires (Coulangeon, p. 27). Malgré tout, cette étude autorise à penser qu'au travers d'œuvres musicales écoutées, l'enseignant d'éducation musicale cherche à transmettre, avant tout, des connaissances déclaratives plutôt spécifiques aux œuvres (notions musicales ou composantes du sonore) dans la mesure où celles-ci sont perceptibles par l'élève, en lien avec l'époque, le genre musical, le programme d'histoire. En effet, même si ce que nous avons appelé "répertoires", en tant que tels, ne sont pas enseignés, les critères et les raisons des choix des enseignants demeurent des candidats pour être enseignés, en tant qu'objets de savoirs explicites. Ils peuvent aussi demeurer à l'état de connaissances implicites mais néanmoins être présents. Pour cela les enseignants sélectionnent des œuvres dans cinq grandes catégories de musiques, essentiellement tonales et modales (c'est nous qui le disons), issues du jazz, des courants populaires et savants du XX ${ }^{\mathrm{e}}$ et du XIX ${ }^{\mathrm{e}}$ siècle, du Romantisme, du Baroque, du Moyen Âge. Ces résultats se situent dans le prolongement des constats faits par Mialaret (2001) : la centration de l'enseignant en termes de transmission de savoirs lors de l'écoute d'œuvres, en classe, se fait sur la perception des notions ou composantes spécifiques à chaque composition ou transversales à plusieurs compositions. En revanche, les enseignants font peu état d'une centration sur le désir, les besoins, la motivation des élèves qui les conduirait à faire des choix correspondants à ce qui est déjà apprécié. Pouvons-nous parler d'une sorte d'accord, de consensus implicite, voire de déférence à l'égard d'un certain nombre de genres et compositeurs qui s'avèrent majoritairement choisis par ces enseignants ? Pour autant, la question du statut de l'œuvre (chef-d'œuvre) ne semble pas du tout préoccuper ces enseignants. Serait-ce une question de valeur conférée à certaines œuvres ? En effet, dans le prolongement de l'enquête de 2005, nous faisions l'hypothèse que les valeurs ont une incidence sur les choix des savoirs à enseigner, autrement dit que la question des valeurs contribue à expliciter des questions d'ordre didactique (Tripier-Mondancin, 2013a, p. 253). La même enquête montrait que les valeurs esthétiques des enseignants interrogés s'enracinent dans des propriétés objectales de l'œuvre (connaissances et savoirs relatifs aux œuvres), tout autant que dans l'interaction artefact-musical et sujet-récepteurmusicien, c'est-à-dire dans la relation esthétique (rôle des émotions et des sentiments dans l'élaboration du jugement de valeur sur l'œuvre). Les résultats de la nouvelle enquête tendraient à renforcer ces résultats.

Ainsi, la musique au collège, en tant que partie prenante du socle commun d'une culture scolaire à la fois explicite et implicite pourrait jouer un rôle, contrairement à ce que certains travaux sociologiques annoncent comme on l'a vu, en confrontant-exposant les élèves à une pluralité de catégories de répertoires musicaux. D'ailleurs, les mêmes travaux sociologiques concluent que l'effet des ressources culturelles et secondairement économiques prévaut sur celui de la socialisation primaire, en regard de la distribution des goûts (Coulangeon, 2003, p. 21) ; l'orientation des préférences relève d'une attitude construite et non pas d'un héritage passif. Dès lors, l'éducation musicale à l'école, en tant qu'institution favorisant la construction de savoirs, conserve donc tout son sens.

À regarder de près l'ensemble de ces résultats, il semble que les enseignants, en termes d'écoute d'œuvres, sont loin d'être seuls pour faire des choix. La didactisation des savoirs démarre déjà à l'université, en musicologie : les musicologues produisent des ouvrages généraux qui opèrent une forme de transposition didactique. Le conservatoire, les médias, internet y participent. Les hypothèses concernant l'influence réciproque entre, certains choix et « l'ancienneté dans le métier » ou encore "l'académie " (selon qu'on est enseignant à Bordeaux ou à Toulouse) sont acceptables. Quant à l'« influence réciproque entre le "genre » (masculin féminin), l'académie et certains critères utilisés pour choisir les œuvres, elle se confirme en partie. Ce que notre enquête met ainsi à jour, c'est, bien plus que le genre, le type de collège, ou l'ancienneté, le rôle, que joue "l'académie » en tant qu'institution au sein de l'institution "Éducation nationale ", en tant qu'intermédiaire entre le ministère, les programmes 
Odile Tripier Mondancin

scolaires, leur mise en ouvre sur le territoire. Ce que recouvre la variable académie mériterait d'être approfondi lors d'une prochaine étude. La formation continuée mise en place et pilotée par les inspecteurs pédagogiques régionaux, dans chaque académie, participe a priori à ce processus de transposition. L'articulation entre les conseillers pédagogiques qui accueillent des étudiants en formation initiale et les formateurs dans les ESPE (École Supérieure du Professorat et de l'Éducation, anciennement IUFM, Instituts Universitaires de Formation des Maîtres) y contribue également largement.

Cette étude, en contribuant à la connaissance des genres et compositeurs écoutés ainsi qu'à la manière dont s'effectuent les choix, participe à l'élaboration d'une didactique de l'écoute en lien avec les questions suivantes : qu'enseigne-t-on, pourquoi ? Les réponses à ces questions, une fois reliées à celle des finalités de l'éducation musicale dans l'école devraient pouvoir servir de point d'appui à la réflexion sur l'élaboration toujours en mouvement d'un parcours d'enseignement-apprentissage durant la scolarité obligatoire.

Se pourrait-il que le rôle joué par l'école impacte la montée de l'éclectisme des goûts, chez les classes dites supérieures, au sens sociologique ? Mais cet éclectisme pourrait aussi être lié, depuis la réforme Haby, à l'élargissement de la base sociale du recrutement des élèves et donc des préférences musicales, élargissement que l'on constate aussi au sein de la classe supérieure, en lien avec la mobilité sociale.

Un autre point de vue serait de dire : la domination symbolique des classes supérieures continue de s'exercer d'une autre manière que dans les années 1970 en lien avec cet éclectisme, par exemple en dignifiant des pratiques jugées jusque-là indigne, comme le jazz esthétisé peu à peu en genre que l'on écoute, au lieu d'un genre sur lequel on danse. Se pourrait-il que cette domination soit favorisée par l'école quand celle-ci facilite l'accès à la pluralité des musiques. Car, il ne faut pas oublier que l'école, tout comme la sociologie, reproduit des nomenclatures de catégories musicales qui font s'opposer savant et populaire, dont le périmètre et la représentation que peuvent en avoir les élèves (ou les répondants des enquêtes d'une manière générale) n'est pas toujours très clair.

Mais l'école peut-elle et doit-elle penser ses missions en dehors d'enjeux sociaux comme les clivages entre les différentes catégories sociales ? L'important n'est-il pas de faire en sorte qu'elle permette à tous les élèves d'acquérir un capital scolaire qui leur permette de construire leur capital culturel ? Dans tous les cas, comme le signale en conclusion Coulangeon, "Le rôle de l'apprentissage sous toutes ses formes, ne peut en être que conforté, ce qui indique l'espace qui demeure ouvert aux politiques de la culture et de l'éducation ». (p. 29).

Nous formulons l'hypothèse " haute » que l'éducation musicale participe, en partie, depuis quelques décennies, à la formation-constitution d'un capital musical minimum qui éventuellement pourrait expliquer l'effet du diplôme sur les écoutes musicales des français.

Une nouvelle enquête dans d'autres académies permettrait d'approfondir la question des œuvres écoutées (et des titres choisis) par niveaux de classe, cette fois. L'analyse que nous menons actuellement sur 23 vidéos de classe devrait nous permettre d'approfondir la question des savoirs enseignés-appris. 


\section{NOTES}

1. Par exemple, cette étude introduit un indicateur de compétence musicale dont la première modalité mériterait de prendre en compte cette forme d'initiation scolaire à la musique : «non musiciens (individus n'ayant aucune formation musicale), personnes ayant suivi une formation musicale (école de musique, conservatoire, cours particulier, etc.), autodidactes (individus ayant appris la musique seuls ou avec des amis) » (Coulangeon. p. 20).

2. Selon Alan F. Chalmers «Les écrits de Popper, Feyerabend et Kuhn abondent en arguments et en exemples à l'appui de la thèse que les observations et les énoncés d'observation dépendent de la théorie. » (Chalmers, p. 72). 3. Savant, au sens de "savoir reconnu culturellement et socialement comme savant par une institution » dès lors l'acception du terme dépasse les débats récurrents : Chevallard introduit l'idée en 1994 que « tout savoir est, pour l'acteur, d'abord «savoir en acte». Tout savoir est pratique sociale» (Chevallard, 1994, cité par Bourg, 2011, p. 33-34).

4. Chant grégorien (42\%), introït, Kyrie, gloria, credo, communion, offertoire, sanctus (22\%), messe polyphonique (14\%), organum (10\%).

5. Messe (10\%).

6. Oratorio (15\%), Messe (11\%).

7. Concertos grosso, pièces de clavecin, préludes et fugues, suites, ouvertures...

8. Opéras, comédies ballets, ballet de cour, oratorios, cantate, messe...

9. Symphonie, musique de chambre, quatuor, ouverture, variations, sonate...

10. Symphonie, musique à programme, poème symphonique, concerto, valses, musique de chambre, variations, ballet romantique...

\section{RÉFÉRENCES}

Anderson, L. W., Krathwohl, D. R., Airasian, P. W., Cruikshank, K. A., Mayer, R. E., Pintrich, P. R., et al. (2001). A taxonomy for learning, teaching, and assessing : revision of Bloom's taxonomy of educational objectives. New-York : Longman.

Astolfi, J.-P., (1994). L'école pour apprendre (3e édition). Paris : ESF

Aubert, L. (2003). Les cultures musicales dans le monde : traditions et transformations. Dans J.-J. Nattiez (dir.), Musiques. Une encyclopédie pour $\mathrm{XXI}^{\mathrm{e}}$ siècle. 3. Musiques et cultures (vol. 3, p. 39-197). Arles, Paris : Actes Sud, Cité de la musique.

Bosseur, J.-Y., (1996). Vocabulaire de la musique contemporaine, Paris : Minerve.

Bourg, A. (2008). Didactique de la musique : apports d'une approche comparatiste. Étude des notions de transposition didactique et d'organisation praxéologique pour l'enseignement/apprentissage du piano. Éducation et didactique, 2 (1), 69-88.

Bourg, A. (2011). Propositions pour la recherche en didactique de la musique. Dans P. Terrien \& J.-L. Leroy (dir.), Perspectives actuelles de la recherche en éducation musicale (p. 29-44). Paris : L'Harmattan.

Bras, J.-Y., (2003). Les courants musicaux du XX siècle. Ou la musique dans tous ses états. Genève : Papillon.

Canguilhem, P., Ferrand, F., (2005). Guide de la musique de la Renaissance. Paris : Fayard.

Caron, S., (2003). La notion de valeur musicale en contexte liturgique. Dans M. Desroches \& F. Guertin (dir.), Construire le savoir musical. Enjeux épistémologiques, esthétiques et sociaux (p. 47-63). Paris: L'Harmattan.

Chailley, J., (1958). Précis de musicologie, Paris : Presses Universitaires de France.

Chalmers, A., F., (1987). Qu'est-ce que la science? ( $2^{\mathrm{e}}$ édition). Paris: La Découverte.

Chevallard, Y., (1991). La transposition didactique ( $2^{\mathrm{e}}$ édition). Grenoble : La pensée sauvage.

Coulangeon, P. (2003). La stratification sociale des goûts musicaux. Le modèle de la légitimité culturelle en question. Revue française de sociologie, 44, 3-33.

Coulangeon, P., (2010). Sociologie des pratiques culturelles ( $2^{\mathrm{e}}$ édition). Paris : La Découverte.

Dahlhaus, C. (1976). Genre. Dans M. Honegger (dir.), Science de la musique (vol. 1). Paris : Bordas.

Damasio, A. R., (1999). Le sentiment même de soi : corps, émotions, conscience (traduit par C. L. et C. Tiercelin), Paris : Odile Jacob.

Delcambre, I. (2007). Contenus d'enseignement et d'apprentissage. Dans Y. Reuter, \& C. Cohen-Azria, \& B. Daunay, \& I. Delcambre, \& D. Lahanier-Reuter (dir.), Dictionnaire des concepts fondamentaux des didactiques (p. 45-51). : De Boeck Université.

Denizeau, G., (1997). Les genres musicaux : vers une nouvelle histoire de la musique. Paris : Larousse.

Denizeau, G., (2005). Guide de la musique : une initiation par les œuvres. Paris: Larousse. 
Denizeau, G., (2010). Les grands compositeurs. Paris : Larousse.

Depelteau, F., (2011). La démarche d'une recherche en sciences humaines. De la question de départ à la communication des résultats (nouvelle édition). Bruxelles : De Boeck Université.

Develay, M., (dir.) (1995). Savoirs scolaires et didactiques des disciplines : une encyclopédie pour aujourd'hui. Paris : ESF.

Donnat, O., (1998). Les pratiques culturelles des français. Enquête 1997 : La documentation française, ministère de la Culture et de la Communication, DEP.

Ferrand, F., (dir.) (1999). Guide de la musique du Moyen Âge; Paris : Fayard.

Goubault, C., (2000); Vocabulaire de la musique à l'aube du $\mathrm{XX}^{\mathrm{e}}$ siècle, Paris : Minerve.

Goubault, C., (1997). Vocabulaire de la musique Romantique. Paris : Minerve.

Heinich, N., (2005). L'élite artiste. Excellence et singularité en régime démocratique. Paris : Gallimard.

Howell, D. C., (1998). Méthodes statistiques en sciences humaines. Paris : De Boeck Université.

Kinnear, P., \& Gray, C., (2005). SPSS facile appliqué à la psychologie et aux sciences sociales. Maîtriser le traitement des données. Bruxelles: De Boeck.

Lemaître, E., (dir.), (1992). Guide de la musique sacrée et chorale profane. L'âge baroque 1600-1750. Paris : Fayard.

Maizières, F., (accepté à l'évaluation, 10 mars 2013). Lécoute musicale à l'école primaire en France : les œuvres déclarées être enseignées, Revue des Sciences de l'éducation.

Massin, J., \& Massin, B., (dir.) (1985), Histoire de la musique occidentale ( $2^{\mathrm{e}}$ édition). Paris : Fayard.

Massip, C., (2007). Le livre de musique. Paris : Bibliothèque nationale de France.

Mialaret, J.-P. (2001). Vers une didactique de l'écoute musicale à l'école. Jalons introductifs. Dans M. Imberty (dir.), De l'écoute à l'œuvre. Études interdisciplinaires (p. 99-112). Paris : L'Harmattan.

Michels, U., (1985). Guide illustré de la musique. Paris : Fayard.

Mili, I. Une maïeutique de l'écoute musicale en classe. Des mots en échos, pour construire l'œuvre musicale. Repères, 43, 153-174. Repéré à http://reperes.revues. org/227.

Ministère de l'éducation nationale. 1938. Programmes. Chant choral, 6e à 3e (Arrêté du 14 avril 1938).

Ministère de l'Éducation nationale. 2008. Programme d'enseignement d'arts plastiques et d'éducation musicale pour les classes de sixième, de cinquième, de quatrième et de troisième du collège (Arrêté du 9 juillet 2008, JO du 5 août 2008, BO spécial $n^{\circ} 6$ du 28 août 2008).

Ministère de l'Éducation nationale .2008. Organisation de l'enseignement de l'histoire des arts à l'école primaire, au collège et au lycée (Arrêté du 11 juillet 2008, J.O. du 27 juillet 2008).
Ministère de l'Éducation nationale .2006. Le socle commun des connaissances et des compétences (décret du 11 juillet 2006).

Musial, M., \& Pradère, F., \& Tricot, A., (dir.) (2012). Comment concevoir un enseignement? Bruxelles : De Boeck.

Noiray, M., (2005). Vocabulaire de la musique de l'époque Classique. Paris : Minerve.

Popper, K. R., (1991), La connaissance objective. Une approche évolutionniste. ( $1^{\text {re }}$ édition : 1979, traduit par J.-J. Rosat). Paris : Flammarion.

Reboul, O., (2010). Qu'est-ce qu'apprendre? Pour une philosophie de l'enseignement ( $1^{\text {re }}$ édition : 1980). Paris : Presses Universitaires de France.

Rice, T. (2005). Est-il possible d'écrire l'histoire des musiques de tradition orale ? ( $1^{\text {re }}$ édition : 2003) Dans J.-J. Nattiez (dir.), Musiques. Une encyclopédie pour le $\mathrm{XXI}^{\mathrm{e}}$ siècle. Tome 3. Musiques et cultures (vol. 3, p. 137162). Paris : Actes Sud, Cité de la Musique.

Snyders, G. (2012). Entretien. La musique comme joie à l'école. Éduquer, Former. Musique et pratiques actuelles en milieu scolaire ( $1^{\text {re }}$ édition : 1999-2000). 43, 153160.

Tripier-Mondancin, O. (2008). L'éducation musicale au collège depuis 1985 : valeurs déclarées par des enseignants d'éducation musicale, genèse. Thèse de doctorat, université Toulouse 2, Toulouse.

Tripier-Mondancin, O. (2010). Changes in Conceptions of Music Education in Junior High School Curricula during the Twentieth Century in France. IJCDSE (International Journal for Cross-Disciplinary Subjects in Education), 1(1), 43-52.

Tripier-Mondancin, O., (2010). L'éducation musicale dans le secondaire. Attributs, formation des enseignants, instructions et programmes de collège, valeurs. Paris : L'Harmattan.

Tripier-Mondancin, O. (2011). L'éducation au choix, évaluation des compositions musicales. Dans J.-L. Leroy, $\&$ P. Terrien, (dir.), Perspectives actuelles de la recherche en éducation musicale (p. 125-138). Paris : L'Harmat$\tan$.

Tripier-Mondancin, O. (2013a). Valeurs esthético-éthiques des enseignants d'éducation musicale. Dans J. Baillé (dir.), Du mot au concept. Valeur (p. 227-258). Grenoble : PUG, Presses universitaires de Grenoble.

Tripier-Mondancin, O. (2013b). Vers une didactique des pratiques vocales en collège : répertoires déclarés être chantés en éducation musicale au collège. Congrès International 2013. AREF. AECSE. Repéré à http://www.aref2013. univ-montp 2 .fr/cod6/? q=content/090-vers-une-didactique-des-pratiques-vocales-en-coll\%C3\%A8ger\% C3\% A 9pertoires-d\% C3\% A 9 clar\% C 3\% A 9s $\%$ C3\%AAtre-chant\%C3\%A9s-0.

Verret, M., (1975). Le temps des études. Paris : HonoréChampion.

Villemin, D. (2011). Réécouter, réécrire. Évolutions du discours descriptif en classe de troisième. Didactique 
du lexique en éducation musicale. Thèse de doctorat, université Paris Sorbonne, Paris.

Von der Weidt, J.-N., (1992). La musique du Xxe siècle.

Paris : Hachette. 